\title{
Very cold cores in the Taurus Molecular Ring as seen by ISO*
}

\author{
L. V. Tóth ${ }^{1,2}$, M. Haas ${ }^{1, \star \star}$, D. Lemke ${ }^{1}$, K. Mattila ${ }^{3}$, and T. Onishi ${ }^{4}$ \\ 1 Max-Planck-Instutute für Astronomie, Königstuhl, 17, 69117, Heidelberg, Germany \\ 2 Department of Astronomy of the Loránd Eötvös University, Pázmány Péter sétány 1, 1117 Budapest, Hungary \\ ${ }^{3}$ Helsinki University Observatory, PO Box 14 Tähtitorninmäki, 00014 University of Helsinki, Finland \\ ${ }^{4}$ Department of Astrophysics, Nagoya University, Chikusa-ku, Nagoya 464-8602, Japan
}

Received 1 November 2003 / Accepted 19 February 2004

\begin{abstract}
Three prominent cold objects of the Taurus Molecular Ring (TMR) were revealed by our ISOPHOT $200 \mu \mathrm{m}$ map of the south-eastern part of the Heiles Cloud 2 (HCL2) cloud complex. One corresponds to the cyanopolyyne peak region of the TMC-1 ridge, (TMC-1 CP), one is the HCL2-E cloud, and one which we call HCL2-ES lies south of TMC-1. The 200/100 $\mu \mathrm{m}$ colour temperatures and column densities of the three ISOPHOT cold clouds are $\approx 12 \mathrm{~K}$, and $1.2 \pm 0.7 \times 10^{22} \mathrm{~cm}^{-2}$ respectively, as calculated from ISO/IRAS surface brightnesses. As Nagoya-4 $\mathrm{m} \mathrm{C}^{18} \mathrm{O}(1-0)$ spectra show, these are dense molecular clouds with $N(\mathrm{H})>10^{21} \mathrm{~cm}^{-2}$ column density peaks. The ISOPHOT $200 \mu \mathrm{m}$ surface brightness is well correlated with the $\mathrm{C}^{18} \mathrm{O}$ line intensity (corr. coef. $\approx 70 \%$ ). The large dust particle emissivity is found to be increased in the prototypical very dense core TMC-1 CP. As the low linewidths $\left(\Delta v=0.8 \pm 0.2 \mathrm{~km} \mathrm{~s}^{-1}\right)$ indicate, the level of turbulent energy density is $50 \%$ lower in these three clouds than in other clouds of HCL2. Dense cores were identified inside the $\mathrm{C}^{18} \mathrm{O}$ clouds by $\mathrm{NH}_{3}$ measurements with the Effelsberg-100 m telescope and Nobeyama- $45 \mathrm{~m} \mathrm{H}^{13} \mathrm{CO}^{+}$data. The density of the dense cores is $n \geq 1.1 \times 10^{5} \mathrm{~cm}^{-3}$, and their kinetic temperatures are $\$ 10 \mathrm{~K}$, in good agreement with the FIR results. The total molecular gas mass in the gravitationally bound cloud cores of TMC-1 CP and HCL2-E is about $21 M_{\odot}$ and $8 M_{\odot}$ respectively. The cores, TMC-1 CPb and HCL2-Eab are associated with 3 low mass YSO candidate 2MASS point sources, while 35 other low mass YSO candidates are seen elsewhere in TMR south, which we consider as evidence for ongoing low mass star formation.
\end{abstract}

Key words. ISM: clouds - dust, extinction - ISM: individual objects: Taurus clouds - ISM: molecules - ISM: structure stars: formation

\section{Introduction}

The process that transforms gravitationally bound cloud cores into collapsing protostars starts with effective cooling. We present a study of very cold and dense cloud cores in Taurus.

Star formation occurs in dense cores within molecular clouds (e.g. Williams et al. 2000), although the study of such regions was hampered for many years by their very large optical depths at near-infrared and optical wavelengths. It is only since the opening up of the far-infrared and submillimetre regimes that astronomers have been able to study molecular clouds in detail. Understanding the theory of star formation requires a detailed observational determination of the initial conditions of the collapse phase that forms a protostar. The pre-protostellar (or prestellar for short) core phase

Send offprint requests to: L. V. Tóth,

e-mail: lvtoth@mpia-hd.mpg.de

* Based on observations with ISO, an ESA project with instruments funded by ESA Member States (especially the PI countries: France, Germany, The Netherlands and the UK) and with the participation of ISAS and NASA.

$\star \star$ Present address: Astonomisches Institut Ruhr-Universität Bochum, 44780 Bochum, Germany.
(Ward-Thompson et al. 1994) is believed to be the stage of star formation that precedes the formation of a protostar and hence should represent observationally the initial conditions of protostellar collapse. Theory predicts that the core geometry prior to this stage is critical in determining the manner of collapse (e.g. Whitworth et al. 1998).

A region was selected in Taurus to observationally examine the above mentioned processes in dense cold clouds with multiwavelength data. Taurus is one of the coldest dusty regions of nearby star formation. The Taurus clouds are at around $140 \mathrm{pc}$ distance (Elias 1978) or even closer at $126 \mathrm{pc}$ (see recently Hartigan \& Kenyon 2003). The DIRBE-based cold dust temperatures are below $16 \mathrm{~K}$ at some locations in Taurus as found e.g. by Lagache et al. (1998). Heiles (1968) traced cold interstellar gas with $\mathrm{OH}$ detections at positions of high visual extinction. Since then the "Heiles Cloud 2" (HCL 2) identifies the most massive molecular cloud in Taurus as was shown by a large scale $\mathrm{C}^{18} \mathrm{O}$ survey by Onishi et al. (1996). The observed positions of Heiles were marked with letters A to T. As a roadmap of HCL2 we present a deep extinction map resulting from the NIR $H-K$ colour study of the Taurus region by Padoan et al. (2002). The map traces the total 


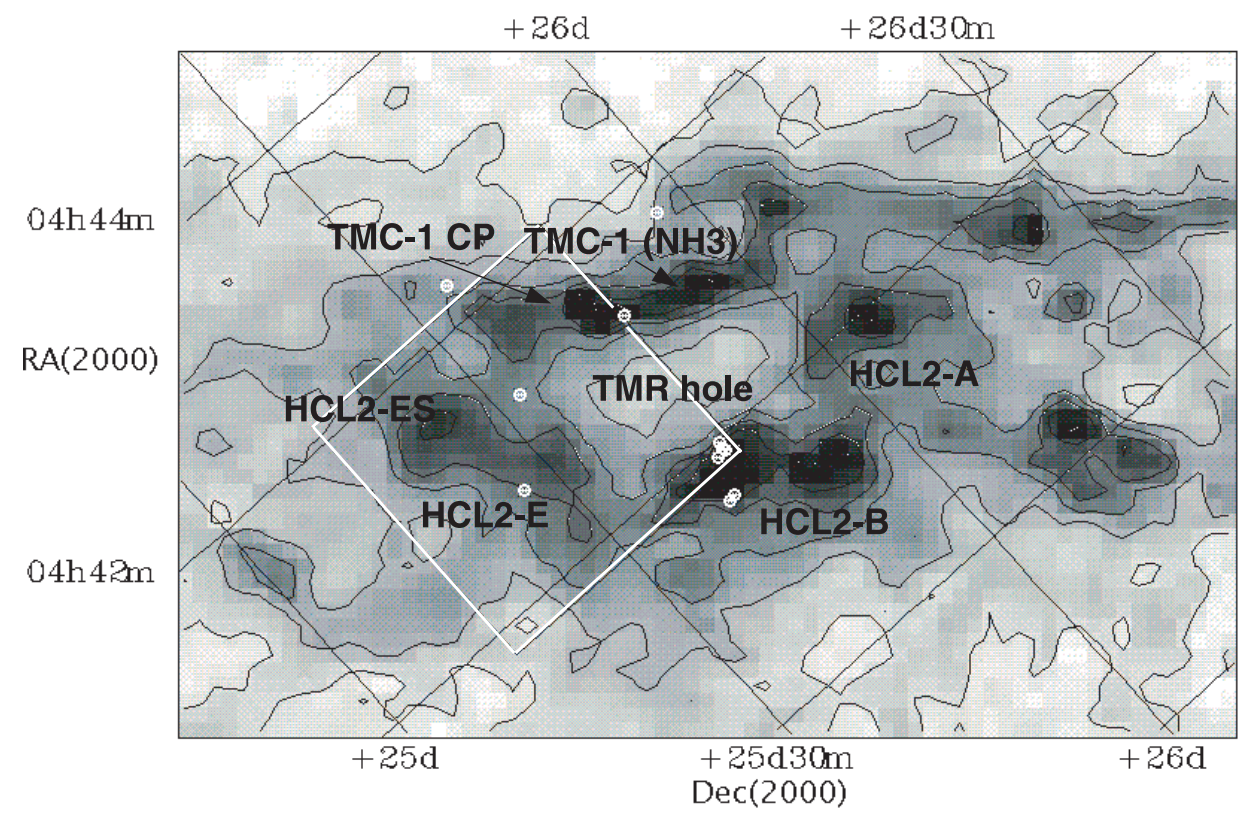

Fig. 1. $A_{\mathrm{V}}$ grayscale map of HCL2 with black contours from 2 mag by 2 mag based on the data of Padoan et al. (2002). Four objects with high peak extinction $\left(A_{\mathrm{V}}\right.$ (peak) $\geq 8 \mathrm{mag}$ ) surround a low extinction region in the centre. These are in clockwise: (1) TMC-1, (2) HCL2-A, (3) HCL2-B and (4) HCL2-E and HCL2-ES, the main clouds of the Taurus Molecular Ring (TMR). The IRAS point sources (see Table 3) are marked with white symbols. The white frame borders the region of our ISOPHOT $200 \mu \mathrm{m}$ map.

ISM column density distribution well up to $10^{22} \mathrm{~cm}^{-2}$. HCL2 breaks up to 4 main clouds (1) TMC-1, (2) HCL2-A, (3) HCL2B and (4) HCL2-E and HCL2-ES, as seen in Fig. 1. While in TMR the visual extinction is $A_{\mathrm{V}}>4 \mathrm{mag}$, it drops in the central hole to the value seen outside HCL2. A schematic view of TMR is given in Fig. 2. Subsequent studies focused mainly on the narrow molecular ridge at the eastern part of HCL2 which is often named just as Taurus Molecular Cloud 1 (TMC-1). The elongated TMC-1 is a unique strong source of emission from carbon chain and other complex molecules. It is fragmented into a chain of at least five large clumps and several small cores (see e.g. Hirahara et al. 1992, or Langer et al. 1995), with a chemical gradient with distinct $\mathrm{C}_{5} \mathrm{H}, \mathrm{HC}_{7} \mathrm{~N}$ and $\mathrm{NH}_{3}$ maxima (Little et al. 1979; Olano et al. 1988). The southeastern part of TMC-1 which is abundant in "carbon-chain" molecules (such as CCS and cyanopolyynes) is also called as "cyanopolyyne peak", hereafter, TMC-1 CP. The northwestern part of the ridge, which is abundant in molecules such as $\mathrm{NH}_{3}$, $\mathrm{N}_{2} \mathrm{H}^{+}$, and SO is often cited as "ammonia peak", hereafter TMC-1 (NH3). It is yet unclear what physical conditions make TMC-1 such a unique laboratory of interstellar chemistry.

Cernicharo et al. (1984) found three of the other opaque sub-clouds of HCL2 to be remarkably similar to TMC-1 with kinetic temperature $T_{\text {kin }}=9 \pm 1 \mathrm{~K}$, and density $n>10^{4} \mathrm{~cm}^{-3}$. Schloerb \& Snell (1984) interpreted the structure of HCL2 based on ${ }^{13} \mathrm{CO}$ and $\mathrm{C}^{18} \mathrm{O}$ data as a $400 M_{\odot}$ rotating ring. They identified 5 gravitationally bound subcondensations in the Taurus Molecular Ring (TMR). Two of the subcondensations roughly correspond to TMC-1, and HCL2-E. The pre-ISO knowledge on the extended FIR emission in Taurus was summarised by Abergel et al. (1994, 1995) however only on large scales. HCL2 appeared at the NW corner of their maps as a bright, cold region.

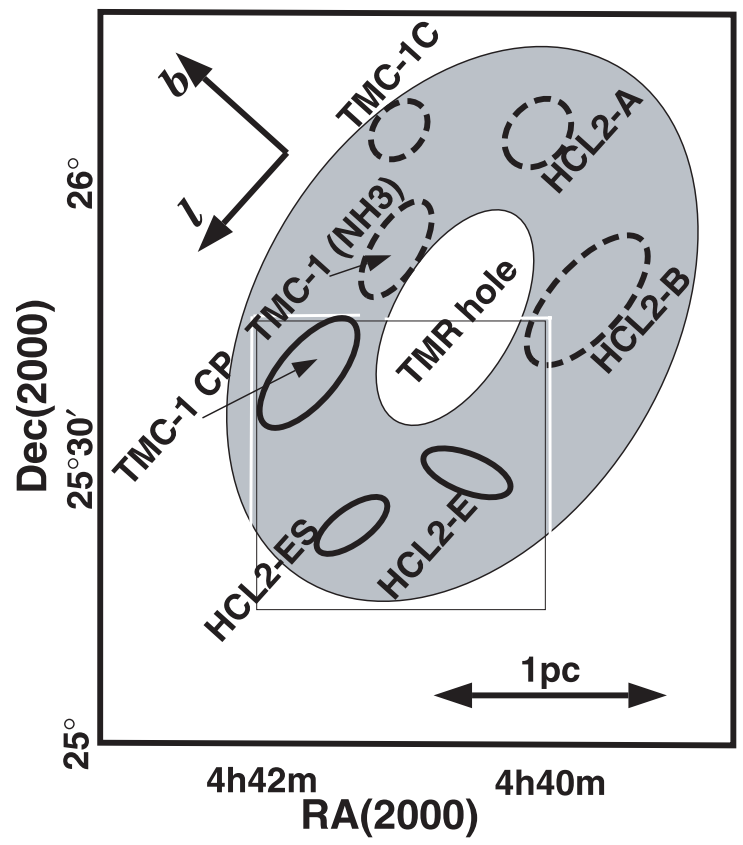

Fig. 2. Roadmap of the TMR clouds. The outer frame corresponds roughly to the extent of HCL2. The shaded elliptical ring follows the $A_{\mathrm{V}}=4$ mag extinction contours. The subclouds inside TMR are marked with ellipses. The 3 clouds inside the ISO $200 \mu \mathrm{m}$ image frame (box) are drawn with solid, the clouds outside with dashed lines. The TMC- $1 \mathrm{NH}_{3}$ peak HCL2 and TMR are elongated parallel to the galactic plane, but not all the dense subclouds follow that orientation.

We present a FIR study of TMC-1 and the southern, low star formation activity part of TMR. We investigate a full sampled $\mathrm{C}^{18} \mathrm{O}$ spectral line map zoomed into HCL2 and TMR. We derive physical properties of TMR and embedded very cold 
cloud cores based on FIR data, $\mathrm{cm}$ and mm spectroscopic data and discuss the conditions for star formation.

\section{Observations and data reduction}

FIR observations were made using ISOPHOT's (Lemke et al. 1996) raster mapping (AOT P22) centered on to $\operatorname{RA}(2000)=4^{\mathrm{h}} 41^{\mathrm{m}} \operatorname{Dec}(2000)=25^{\circ} 30^{\prime}$, during orbit 862 on 1998 March 26 . A $30^{\prime} \times 30^{\prime}$ field in HLC2 was mapped with the PHT-C200 camera associated with the $C-200$ filter, which has a reference wavelength of $200 \mu \mathrm{m}$ and a bandwidth fullwidth at half-maximum $(F W H M)$ of $30 \mu \mathrm{m}$. The $200 \mu \mathrm{m}$ map covers the southern part of TMR: the cyanopolyyne maximum region of the TMC-1 ridge (hereafter TMC-1 CP), the opaque cloud at HCL2-E, and a small part of HCL2-B. (see Figs. 9 and $3 a)$.

We assume a calibration accuracy of $30 \%$ for the ISOPHOT data (see Klaas et al. 1998, 2002). The data were reduced using the version 11 of the PHT Interactive Analysis (PIA) software (Gabriel et al. 1997). We followed the standard pipeline data reduction procedure, but used our own routines for the flatfield correction. We note that the oversampled mapping mode (AOT P32) $200 \mu \mathrm{m}$ data of TMC-1 CP, as given in the archive (TDT No. 68502362), show less than $10 \%$ difference from our values.

High Resolution IRAS Galaxy Atlas (IGA) images at 60 and $100 \mu \mathrm{m}$ (Cao et al. 1997) were used to identify uncatalogued IRAS point sources and for a comparison with the ISO $200 \mu \mathrm{m}$ surface brightness data. The $100 \mu \mathrm{m}$ IGA image was calibrated with a corresponding SFD $100 \mu \mathrm{m}$ image (Schlegel et al. 1998) The SDF $100 \mu \mathrm{m}$ images are derived from ISSA data: zodiacal foreground and confirmed point sources were removed, as were artifacts from the IRAS scan pattern were removed. The result of all these manipulations is a $100 \mu \mathrm{m}$ surface brightness map with DIRBE-quality calibration and $\approx 2.5^{\prime}$ resolution. (see Fig. 3 )

CO $2.6 \mathrm{~mm}$ line observations of TMR were taken with $2^{\prime}$ spacing and a resolution of $H P B W=2.7$ as part of the NagoyaNanten CO survey of the Milky Way. Detailes of the Nagoya$4 \mathrm{~m}$ measurements are given in Onishi et al. $(1996,1998)$. Figure 4 displays average $\mathrm{C}^{18} \mathrm{O}(1-0)$ spectra of the 3 regions marked in Fig. 3 and of the TMR hole.

$\mathrm{NH}_{3}(1,1)$ and $(2,2) 1.3 \mathrm{~cm}$ line observations were carried out in 2002 March, 2002 Sept., and 2003 Feb. with the Effelsberg-100 $\mathrm{m}$ radiotelescope to trace dense gas associated with the cores, and to derive kinetic temperatures. The HPBW at the observing frequency of $23.7 \mathrm{GHz}$ is $40^{\prime \prime}$. The facility $1.3 \mathrm{~cm}$ receiver was used with a typical system temperature (antenna temperature units) on the sky of $180 \mathrm{~K}$, $150 \mathrm{~K}$, and $120 \mathrm{~K}$ for the three observing runs respectively. The backend was the facility 8096 channel autocorelator split into 4 bands in order to observe simultaneously both polarizations at the $\mathrm{NH}_{3}(1,1)$ and $(2,2)$ rest frequencies of $23694.495 \mathrm{MHz}$ and $23722.633 \mathrm{MHz}$, respectively. The resulting spectral resolution was $0.06 \mathrm{~km} \mathrm{~s}^{-1}, 0.12 \mathrm{~km} \mathrm{~s}^{-1}$, and $0.06 \mathrm{~km} \mathrm{~s}^{-1}$ for the three observing runs respectively. The ISOPHOT cold cloud at the HCL2-E position was mapped with a resolution of $40^{\prime \prime}$ and the $T(1,1)$ peak region with $28^{\prime \prime}$ resolution. Spectra at the $\mathrm{NH}_{3}(1,1)$ maxima of HCL2-E are shown in Fig. 5.

Pointing and calibration were checked by continuum scans across 3C 123 3C 138 and NGC 7027. We estimate a pointing accuracy of $5^{\prime \prime}$. We calibrated our data assuming a main beam brightness temperature of NGC 7027 of $8.03 \mathrm{~K}$ coresponding to $5.58 \mathrm{Jy}$ at $1.3 \mathrm{~cm}$ (Peng et al. 2000). We confirmed our calibration and velocity scale observing also $\mathrm{TMC}-1 \mathrm{CP}$ at RA $(2000)=4^{\mathrm{h}} 41^{\mathrm{m}} 42^{\mathrm{s}}, \operatorname{Dec}(2000)=25^{\circ} 41^{\prime} 42^{\prime \prime}$ (see Effelsberg-100 m spectra by Takano et al. 1998). Our $\mathrm{NH}_{3}$ spectra were reduced using CLASS ${ }^{1}$ software, and analysed following procedures described by Harju et al. (1993). The observed $\mathrm{NH}_{3}$ line parameters, and derived quantities are listed in Table 2. where the columns are: (1) position, (2) line, (3) main beam brightness temperature, (4) velocity $v_{\mathrm{LSR}}$, (5) line-width from single Gaussian fit to the main group line, (6) line-width from hyperfine fit, (7) main group line area, (8) total line area of satellites, (9) optical depth. We note that the parameters at HCL2E $(-240,-60)$ are derived from average spectra in a $90^{\prime}$ diameter region.

\section{Results}

\subsection{Far-infrared data}

Morphology on FIR maps: Figures $3 \mathrm{a}-\mathrm{c}$, show the ISOPHOT $200 \mu \mathrm{m}$, IRAS $100 \mu \mathrm{m}$, and IRAS $60 \mu \mathrm{m}$ surface brightness distribution in the southern part of HCL2. The $200 \mu \mathrm{m}$ bright regions outline the Taurus Molecular Ring (TMR). We define five regions on the $200 \mu \mathrm{m}$ image for our further studies: (i) TMC-1 CP; (ii) HCL2-E; (iii) HCL2-ES; (iv) TMR hole; (v) TMR. TMC-1 CP, HCL2-E, and HCL2-ES are the 3 local maxima on the $200 \mu \mathrm{m}$ image. We define TMR as the region where $I_{200}>140 \mathrm{MJy} \mathrm{sr}^{-1}$. Coordinates and derived parameters are listed in Table 1. The brightest FIR object of $12^{\prime} \times 6^{\prime}$ size at $\operatorname{RA}(2000)=4^{\mathrm{h}} 41^{\mathrm{m}} 34.97, \operatorname{Dec}(2000)=+25^{\circ} 38^{\prime} 53^{\mathrm{s}} .2$ with an intensity maximum of $210 \mathrm{MJy} \mathrm{sr}^{-1}$ corresponds to TMC-1 CP, the $\mathrm{HC}_{3} \mathrm{~N}(J=1-0, F=2-1)$ integrated line intensity peak region $\left(W\left(\mathrm{HC}_{3} \mathrm{~N}\right)>0.3 \mathrm{~K} \mathrm{~km} \mathrm{~s}^{-1}\right.$ by Tölle et al. 1981) of TMC-1. The southern part of TMR appears as a 24' size diffuse cloud with two maxima, one is at HCL2-E and one lies SE of it. Each of these have sizes similar to the TMC-1 CP core. The edge of the HCL2-B star forming cloud shows in at the NW corner of the image. The $98 \mathrm{MJy} \mathrm{sr}^{-1}$ intensity minimum of our ISOPHOT $200 \mu \mathrm{m}$ image (Fig. 3a) occurs at the dark central hole of TMR.

TMR is bright in the IRAS $100 \mu \mathrm{m}$ image (Fig. 3b) as well; however, it peaks only at HCL2-B. A large diffuse cloud appears associated with the other $200 \mu \mathrm{m}$ bright cloudlets, with a $100 \mu \mathrm{m}$ maximum between them. The $100 \mu \mathrm{m}$ surface brightness of the TMR hole appears to be as low as the value outside TMR.

The $60 \mu \mathrm{m}$ IRAS image (Fig. 3c) shows sharp peaks at the northern edge, namely the SE boundary of HCL2-B with a group of low mass YSOs and IRAS 04381+2540 a protostellar object (Brown \& Chandler 1999) in TMC-1. Three further

\footnotetext{
1 A GILDAS working group software.
} 

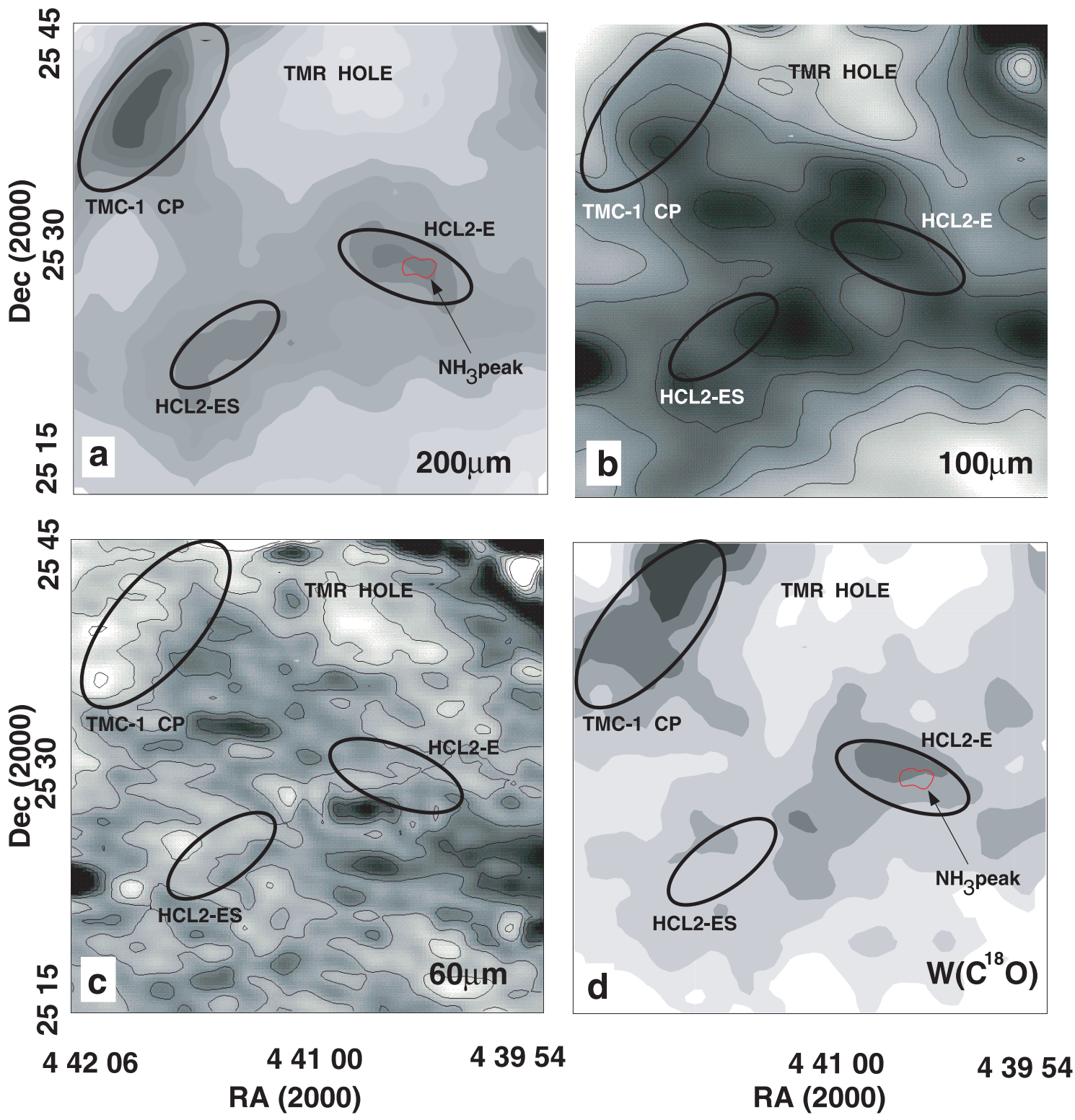

Fig. 3. The southern part of the Taurus Molecular Ring (TMR) with the 3 main clouds (black ellipses) and the TMR hole in the multiwavelengh data. a) ISOPHOT $200 \mu \mathrm{m}$ surface brightness $I_{200}\left(H P B W=2^{\prime}\right)$, greyscale levels: from $100 \mathrm{MJy} \mathrm{sr}^{-1}$ to $190 \mathrm{MJy} \mathrm{sr}^{-1}$ the $\mathrm{NH}_{3}(1,1)$ maximum at HCL2E is marked with a red contour at HCL2-E $\left(H P B W=2^{\prime}\right)$; b) IRAS $100 \mu$ m intensity $I_{100}\left(H P B W=4^{\prime}\right)$ contours: from $25 \mathrm{MJy}^{-1}$ by $1.5 \mathrm{MJy} \mathrm{sr}^{-1}$; c) IRAS $60 \mu \mathrm{m}$ intensity $I_{60}\left(H P B W=4^{\prime}\right)$ contours: from $2.5 \mathrm{MJy} \mathrm{sr}^{-1}$ by $0.3 \mathrm{MJy} \mathrm{sr}^{-1}$; d) $\mathrm{C}^{18} \mathrm{O}$ line intensity $W\left(\mathrm{C}^{18} \mathrm{O}\right)$ $\left(H P B W=2\right.$ '.7), greyscale levels: from $1 \mathrm{~K} \mathrm{~km} \mathrm{~s}^{-1}$ to $2 \mathrm{~K} \mathrm{~km} \mathrm{~s}^{-1}$. The parameters of the marked regions are listed in Table 1 .

Table 1. Parameters of 3 clouds of the southern part of TMR, TMR hole, and TMR (see Fig. 3 ) Notes: TMR and the TMR hole are approximately two times larger than the part seen in our ISOPHOT image. The $\Delta I_{100}$ and $\Delta I_{200}$ excess surface brightness values are relative to the TMR hole. The FIR colour temperature of the TMR hole was calculated from the absolute surface brightness values.

\begin{tabular}{|c|c|c|c|c|c|c|c|c|c|c|}
\hline Region & $\mathrm{RA}(2000)$ & $\operatorname{Dec}(2000)$ & $\begin{array}{c}I_{60}, I_{100}, I_{200} \\
\mathrm{MJy} \mathrm{sr}^{-1}\end{array}$ & $\begin{array}{c}\Delta I_{100}, \Delta I_{200} \\
\mathrm{MJy} \mathrm{sr}^{-1}\end{array}$ & $\begin{array}{l}T_{\mathrm{d}} \\
\mathrm{K}\end{array}$ & $\begin{array}{l}d_{\mathrm{eff}} \\
\mathrm{pc}\end{array}$ & $a / b$ & $\begin{array}{c}\Delta v \\
\mathrm{~km} \mathrm{~s}^{-1}\end{array}$ & $\begin{array}{c}N\left(\mathrm{H}_{2}\right) \\
10^{21} \mathrm{~cm}^{-2}\end{array}$ & $\begin{array}{c}M \\
M_{\odot}\end{array}$ \\
\hline TMC-1 CP & $4^{\mathrm{h}} 41^{\mathrm{h}} 35^{\mathrm{s}}$ & $+25^{\circ} 38^{\prime} 53^{\prime \prime}$ & $3.0, \quad 30.1, \quad 193$ & $5.0,88$ & $11.4 \pm 1.3$ & 0.27 & 2.0 & 0.8 & 18 & 26.8 \\
\hline HCL 2-E & $\begin{array}{lll}4 & 41 & 01\end{array}$ & +253032 & $3.4, \quad 32.4, \quad 176$ & $7.2, \quad 71$ & $12.6 \pm 1.3$ & 0.22 & 2.2 & 1.0 & 11 & 15.0 \\
\hline HCL 2-ES & $4 \quad 40 \quad 33$ & +253025 & $3.2,32.5,173$ & $7.5, \quad 68$ & $12.7 \pm 1.3$ & 0.18 & 2.5 & 0.5 & 11 & 7.4 \\
\hline TMR hole & $4 \quad 40 \quad 41$ & +254321 & $3.0, \quad 25.2, \quad 105$ &,$- \quad-$ & $14.8 \pm 1.0$ & 0.24 & - & 0.9 & 4.5 & - \\
\hline TMR south & $\begin{array}{lll}4 & 40 & 41\end{array}$ & +253000 & $3.4, \quad 30.7, \quad 159$ & $5.6, \quad 54$ & $12.6 \pm 1.5$ & 1.2 & - & 0.8 & 11 & 245 \\
\hline
\end{tabular}


Table 2. Parameters of the ammonia spectra measured.

\begin{tabular}{lccccccccccc}
\hline \hline Position & Line & $\begin{array}{c}T_{\mathrm{MB}}(\mathrm{main}) \\
\mathrm{K}\end{array}$ & $\begin{array}{c}v_{\mathrm{LSR}} \\
\mathrm{km} \mathrm{s}^{-1}\end{array}$ & $\begin{array}{c}F W H M(\text { main }) \\
\mathrm{km} \mathrm{s}^{-1}\end{array}$ & $\begin{array}{c}F W H M \\
\mathrm{~km} \mathrm{~s}^{-1}\end{array}$ & $\begin{array}{c}\text { Areamain } \\
\mathrm{K} \mathrm{km} \mathrm{s}^{-1}\end{array}$ & $\begin{array}{c}\text { Areasatel } \\
\mathrm{K} \mathrm{km} \mathrm{s}^{-1}\end{array}$ & $\begin{array}{c}\tau \\
T_{\text {kin }} \\
\mathrm{K}\end{array}$ & $\begin{array}{c}N\left(\mathrm{H}_{2}\right) \\
\mathrm{cm}^{-2}\end{array}$ & $\begin{array}{c}n\left(\mathrm{H}_{2}\right) \\
\mathrm{cm}^{-3}\end{array}$ \\
\hline TMC-1 CP & $(1,1)$ & $2.40(0.03)$ & $5.90(0.1)$ & $0.72(0.03)$ & $0.54(0.04)$ & $1.68(0.02)$ & $2.0(0.02)$ & $0.5(0.1)$ & $9.4_{-1}^{+1}$ & $1.1 \mathrm{e}+23$ & $4.0 \mathrm{e}+6$ \\
TMC-1 CP & $(2,2)$ & $0.12(0.03)$ & $5.90(0.1)$ & $0.50(0.10)$ & - & $0.07(0.02)$ & - & - & & \\
HCL2E (-240,-60) & $(1,1)$ & $0.99(0.07)$ & $4.68(0.01)$ & $0.77(0.03)$ & $0.50(0.02)$ & $0.96(0.03)$ & $0.81(0.05)$ & $0.4(0.2)$ & $10.3_{-2}^{+1}$ & $4.2 \mathrm{e}+22$ & $2.6 \mathrm{e}+05$ \\
HCL2E (-240,-60) & $(2,2)$ & $0.07(0.03)$ & $4.70(0.10)$ & $1.20(0.30)$ & - & $0.09(0.02)$ & - & - & & \\
HCL2E (-180,-100) & $(1,1)$ & $1.15(0.09)$ & $4.65(0.02)$ & $0.77(0.04)$ & $0.49(0.03)$ & $0.94(0.04)$ & $1.0(0.1)$ & $0.7(0.3)$ & $14.8_{-2}^{+1}$ & $3.0 \mathrm{e}+22$ & $2.3 \mathrm{e}+05$ \\
HCL2E $(-180,-100)$ & $(2,2)$ & $0.26(0.06)$ & $4.65(0.05)$ & $0.57(0.09)$ & - & $0.15(0.02)$ & - & - & & & \\
\hline
\end{tabular}
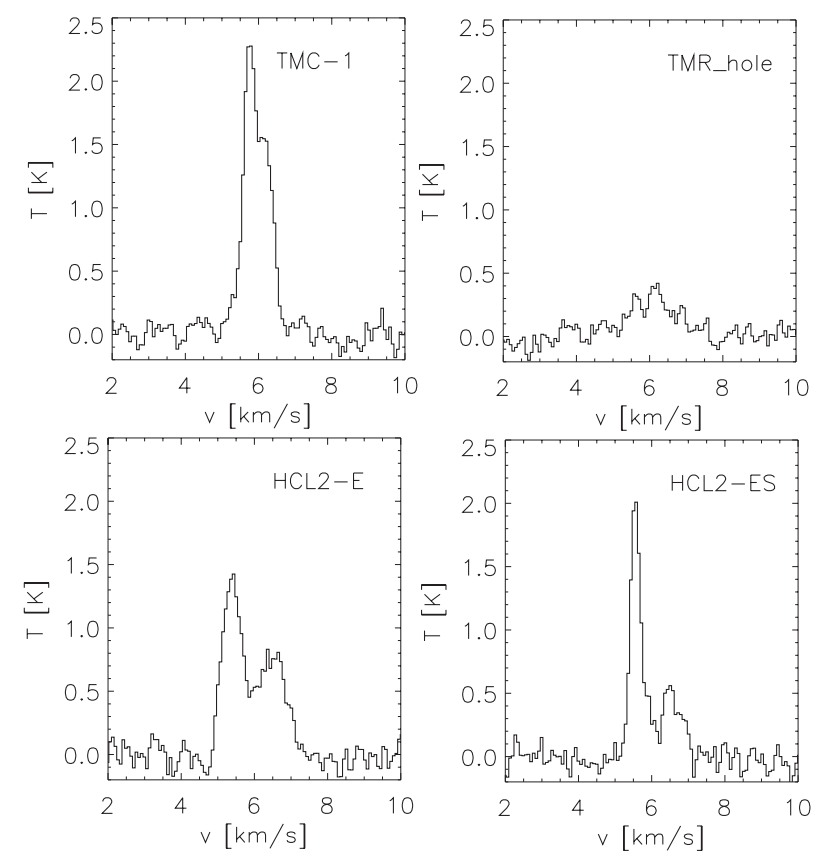

Fig. 4. $\mathrm{C}^{18} \mathrm{O}(1-0)$ spectra at the 4 regions marked in Fig. 3. a) TMC$1 \mathrm{CP}$ at $l=174.40^{\circ}, b=-13.49^{\circ}$. b) HCL2-E at $l=174.35^{\circ}, b=$ $-13.77^{\circ}$. c) HCL2-ES at $l=174.60^{\circ}, b=-13.67^{\circ}$. d) TMR hole at $l=174.20^{\circ}, b=-13.60^{\circ}$.

diffuse local maxima are seen in between TMC-1 CP, east of HCL2-ES, and west of HCL2-E.

FIR colours and column densities of the ISOPHOT cores and of TMR: There is very little variation of the $60 \mu \mathrm{m}$ surface brightness $I_{60}$ seen in the $1 / 4$ square degree field. All 5 regions we defined have an average $I_{60}=3.5 \pm 0.1 \mathrm{MJy} \mathrm{sr}^{-1}$, only those pixels are slightly brighter which correspond to the IRAS point sources of Table 3. The low level and little variation of $I_{60}$ indicate a very low contribution of warm dust, this allows us to interpret the $I_{200}$ vs. $I_{100}$ colour as an indicator of dust temperature.

The weak correlation between the $I_{200}$ and $I_{100}$ images reflects a variation of colour temperature (see Figs. 3 and 7). As the ratio of $I_{200}$ to $I_{100}$ indicates, the low density regions of HCL2, like the TMR hole have the characteristic temperature of the cold galactic clouds, i.e. $\approx 15 \mathrm{~K}$ (see e.g., Lagache et al. 1998, using COBE DIRBE). The southern edge of the star forming HCL2-B appears warm, while TMR south and the
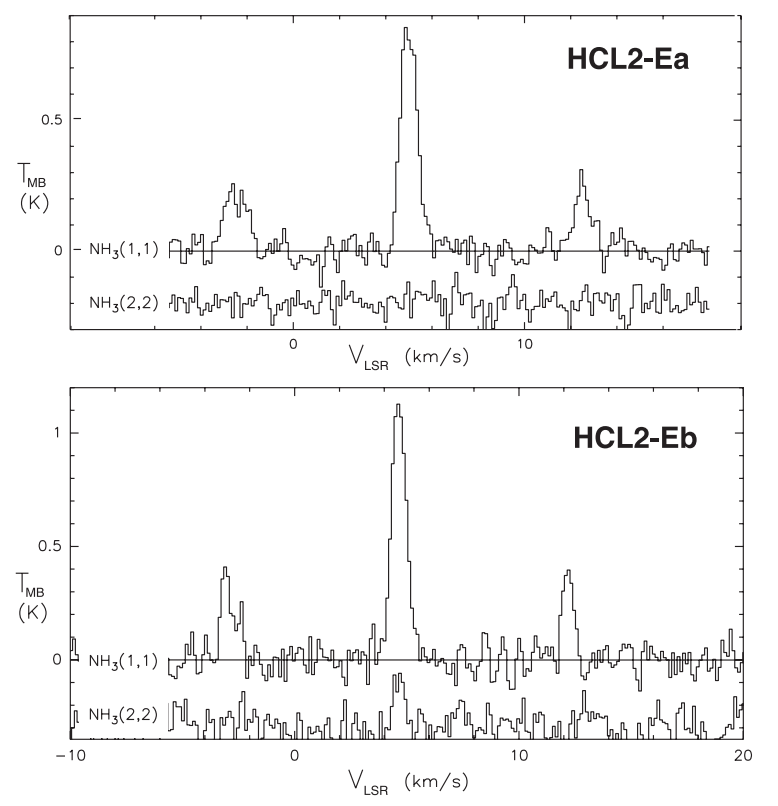

Fig. 5. a) Effelsberg-100 $\mathrm{m} \mathrm{NH}_{3}(1,1)$ and $(2,2)$ spectra at HCL2-Ea the $(1,1)$ main group line area peak of HCL2-E (see Fig. 3). b) Effelsberg-100 $\mathrm{m} \mathrm{NH}_{3}(1,1)$ and $(2,2)$ spectra at the HCL2-Eb; the $(2,2)$ peak position of HCL2-E. (The middle part of the spectra is presented.)

cloudlets HCL2-E and HCL2-ES are cold. TMC-1 is clearly colder than the rest of TMR as seen in Fig. 7b.

Since both the extinction (see Fig. 1) and emission (see Fig. 3) of the dust of the TMR hole look similar to those of the region outside TMR, we define "background" values of FIR surface brightnesses at the TMR hole. The average surface brightness values at the TMR hole were subtracted accordingly, deriving background/foreground corrected 200, 100 and $60 \mu \mathrm{m}$ surface brightnesses $\Delta I_{200}$ and $\Delta I_{100}$. The $\Delta I_{200}$ and $\Delta I_{100}$ were used to calculate the physical parameters of cold dust in TMR and in its clouds. TMC- $1 \mathrm{CP}$ with $T_{\mathrm{d}}=11.4 \pm 0.5 \mathrm{~K}$ is one of the coldest clouds in the Milky Way. The apparently weak contribution of warm dust radiation to the $I_{100}$ surface brightness was estimated applying an empirical $\Delta I_{100}($ warm $)=5 \Delta I_{60}$ formula. When the $\Delta I_{100}$ values are corrected substracting $\Delta I_{100}$ (warm) the colour temperatures become approximately $1 \mathrm{~K}$ lower than listed in Table 1.

Column densities were estimated from $\Delta I_{200}$ and $\Delta I_{100}$ assuming an opacity $\tau \propto v^{2}$ following Juvela et al. (2002), $N(\mathrm{H})_{\mathrm{TMC}-1}=2 \times 10^{22} \mathrm{~cm}^{-2}$ and $N(\mathrm{H})_{\mathrm{TMR}}=0.7 \times 10^{22} \mathrm{~cm}^{-2}$ 
Table 3. IRAS point sources of TMR south. The first 4 sources are inside the region of the ISOPHOT frame, the rest are $\approx 1^{\prime}$ outside the field, but still seen at the boundary due to the IRAS point spread function.

\begin{tabular}{|c|c|c|c|c|c|c|c|}
\hline Source & $\begin{array}{l}\text { RA } \\
(2000)\end{array}$ & $\begin{array}{l}\text { Dec } \\
(2000)\end{array}$ & $\begin{array}{l}F_{12} \\
\mathrm{Jy}\end{array}$ & $\begin{array}{l}F_{25} \\
\mathrm{Jy}\end{array}$ & $\begin{array}{c}F_{60} \\
\text { Jy }\end{array}$ & $\begin{array}{c}F_{100} \\
\text { Jy }\end{array}$ & Other name, classification and reference for class \\
\hline IRAS $04377+2518$ & $04^{\mathrm{h}} 40^{\mathrm{m}} 37^{\mathrm{s}} .9$ & $+25^{\circ} 23^{\prime} 53^{\prime \prime}$ & & & $0.7(.1)$ & & $60 \mu \mathrm{m}$ only point source, this paper \\
\hline IRAS $04378+2521$ & $04^{\mathrm{h}} 40^{\mathrm{m}} 42.6$ & $+25^{\circ} 27^{\prime} 21^{\prime \prime}$ & 1.1(.05) & $1.1(.1)$ & $0.7(.1)$ & & HCL2-SE 2, T Tauri type, this paper \\
\hline IRAS $04382+2527$ & $\begin{array}{lll}04 & 41 & 17.2\end{array}$ & +253252 & & & & $3.1(0.5)$ & Cirrus knot, Harju et al. (2001) \\
\hline IRAS $04379+2534$ & $\begin{array}{lll}04 & 41 & 03.1\end{array}$ & +253947 & $0.44(.04)$ & & & & TNS87 12, star, Tamura et al. (1987) \\
\hline IRAS $04381+2540$ & $\begin{array}{lll}04 & 41 & 12.5\end{array}$ & +254637 & $0.44(.06)$ & $2.7(.2)$ & $10.3(.8)$ & $13.9(1.5)$ & Protostar at the edge of TMC-1, Brown \& Chandler (1999) \\
\hline IRAS $04369+2539$ & $04 \quad 39 \quad 58.4$ & +254506 & $5.0(.2)$ & $6.8(.5)$ & $7.3(.7)$ & $18.2(2.5)$ & IC 2087, refl. nebula, and T Tauri stars, Koenig et al. (2001) \\
\hline IRAS $04390+2517$ & 0442 & +252310 & $0.6(.1)$ & $0.8(.1)$ & $1.6(.2)$ & $7.7(1.4)$ & V955 Tau, T Tauri type, Rydgren \& Vrba (1983) \\
\hline
\end{tabular}

were derived for the peak column density in the TMC-1 CP region and as an average for the whole TMR south respectively.

\subsection{Molecular line data}

The $\mathbf{C}^{\mathbf{1 8}} \mathbf{O}$ spectra of TMR (Fig. 4) show two velocity components of which one is an extended weak component at the velocity of the thin molecular gas traced e.g. by ${ }^{13} \mathrm{CO}$. It peaks at the HCL2-E/HCL2-B region. The strong component is a narrow $\left(\Delta v \approx 0.6 \mathrm{~km} \mathrm{~s}^{-1}\right)$ line, with $T_{a}\left(\mathrm{C}^{18} \mathrm{O}\right)$ maxima at TMC-1 CP and HCL2-ES.

Two main objects can be identified with the $\mathrm{C}^{18} \mathrm{O}$ line intensity $W\left(\mathrm{C}^{18} \mathrm{O}\right)$ distribution in Fig. 3d: TMC-1 (top left) and HCL2-E (centre right). There is also an elongated complex seen including HCLSE-SE, HCL2-E, and HCL2-B. The $W\left(\mathrm{C}^{18} \mathrm{O}\right)$ distribution is well correlated with that of $I_{200}$. The cloudy regions outline TMR. $W\left(\mathrm{C}^{18} \mathrm{O}\right)$ increases with a high gradient $\left(1 \mathrm{~K} \mathrm{~km} \mathrm{~s}^{-1} / 10^{\prime}\right)$ at the outer limits of TMR and drops similarly quickly at the inner cavity. The $\mathrm{C}^{18} \mathrm{O}$ antenna temperature $T\left(\mathrm{C}^{18} \mathrm{O}\right)$ maximum is located inside TMC-1 CP, secondary maxima are seen at the TMC- $1 \mathrm{NH}_{3}$ peak (outside our ISOPHOT map) and in HCL2-ES similarly to the ${ }^{13} \mathrm{CO}(1-0)$ line. All the 3 objects of Fig. 3 have $T\left(\mathrm{C}^{18} \mathrm{O}\right)>1.3 \mathrm{~K}$. While the ${ }^{13} \mathrm{CO}(1-0)$ line intensity $W\left({ }^{13} \mathrm{CO}\right)$ peaks at HCL2-E the $W\left(\mathrm{C}^{18} \mathrm{O}\right)$ maximum is located at the $\mathrm{TMC}-1 \mathrm{NH}_{3}$ peak and secondary maxima are seen at TMC-1 CP, at HCL2-B, at HCL2-E and at HCL2-A (outside the ISO image area).

The $N\left(\mathrm{H}_{2}\right)$ molecular hydrogen column density in TMR and in its sub-clouds was estimated from the $\mathrm{C}^{18} \mathrm{O}$ spectra following Onishi et al. (1996). An excitation temperature of $T_{\text {ex }}\left(\mathrm{C}^{18} \mathrm{O}\right)=10 \mathrm{~K}$, and a $\mathrm{C}^{18} \mathrm{O}$ to $\mathrm{H}_{2}$ abundance ratio of $X\left(\mathrm{C}^{18} \mathrm{O}\right)=10^{7}$ were assumed. The $\mathrm{H}_{2}$ column density ranges from $N\left(\mathrm{H}_{2}\right)=2 \times 10^{21} \mathrm{~cm}^{-2}$ (in TMR hole) to $N\left(\mathrm{H}_{2}\right)=$ $2 \times 10^{22} \mathrm{~cm}^{-2}$ (in TMC-1 CP) with a TMR south average of $N\left(\mathrm{H}_{2}\right) \approx 10^{22} \mathrm{~cm}^{-2}$.

The $\mathbf{N H}_{3}(\mathbf{1}, \mathbf{1})$ line is detected throughout HCL2-E with a brightness temperature $T_{\mathrm{MB}}$ (main) maximum at $\mathrm{RA}(2000)=$ $4^{\mathrm{h}} 40^{\mathrm{m}} 38.4^{\mathrm{s}}, \operatorname{Dec}(2000)=25^{\circ} 29^{\prime} 42^{\prime \prime}$ and line intensity maximum at $\operatorname{RA}(2000)=4^{\mathrm{h}} 40^{\mathrm{m}} 29.5^{\mathrm{s}}, \operatorname{Dec}(2000)=25^{\circ} 29^{\prime} 43^{\prime \prime}$. The $\mathrm{NH}_{3}(1,1)$ velocity is $-0.7 \mathrm{~km} \mathrm{~s}^{-1}$ (blue) shifted relative to the intensity weighted average velocity $v_{\text {moment }}\left(\mathrm{C}^{18} \mathrm{O}\right)$ of the core. The $\mathrm{NH}_{3}(1,1)$ line-width in HCL2-E $\left(\Delta v=0.5 \mathrm{~km} \mathrm{~s}^{-1}\right)$ is similar to that at TMC-1 CP. The boundary of the $\mathrm{NH}_{3}$ core is drawn with the $\mathrm{NH}_{3}(1,1)$ line area contour $W\left(\mathrm{NH}_{3}\right)=0.6 \mathrm{~K} \mathrm{~km} \mathrm{~s}^{-1}$. It apparently coincides with a peak of $I_{200}$. The $\mathbf{N H}_{3}(2,2)$ line was detected at few positions around the $\mathrm{NH}_{3}(1,1)$ peak.

The derived peak density $n\left(\mathrm{H}_{2}\right)=2.6 \times 10^{5} \mathrm{~cm}^{-3}$, and kinetic temperature minimum of $T_{\text {kin }}=10.3_{-1}^{+1} \mathrm{~K}$ indicate that HCL2-E is similar to TMC-1 but less dense and less cold.

\subsection{YSOs and protostars}

FIR point sources of the region were selected from the IRAS Point Source Catalogue, and checking individual IRAS detector snips with Scanpi of the IRSA Server of the JPL (Helou \& Kahn 1986). The sources are listed in Table 3 where the columns are (1) Name which contains RA(1950) and Dec(1950), (2-3) RA(2000) and Dec(2000) coordinates, (4-7) $12,25,60$ and $100 \mu \mathrm{m}$ fluxes respectively (8) classification and reference. The first two sources IRAS $04377+2518$ and IRAS $04378+2521$ were newly identified on the $60 \mu \mathrm{m}$ IGA image, photometry was obtained using Scanpi of IPAC (HELOU et al. 1986). IRAS $04378+2521$ was repeatedly detected by IRAS in 3 bands but the point source template could not be fitted by the Scanpi software. By its FIR colours IRAS $04378+2521$ resembles to an embedded young stellar object (Weintraub 1990). Its total IRAS flux is $F=$ $3.3 \times 10^{-13} \mathrm{~W} \mathrm{~m}{ }^{-2}$ corresponding to $L \approx 0.2 L_{\odot}$, i.e. a YSO with $\approx 0.6 M_{\odot}$. We also obtained a $60 \mu \mathrm{m}$ flux for IRAS $04382+2527$. IRAS $04377+2518$ is a $60 \mu \mathrm{m}$ only source.

NIR point sources as YSOs: There are 804 point sources listed in the new release of the 2MASS point source catalogue (Cutri et al. 2003) within the frame of our ISOPHOT image. A rough classification is possible in the $J-H$ vs. $H-K$ colour-colour diagram (see Fig. 6a). As many as 181 of these are located in the locus of classical T Tauris (Meyer et al. 1997) i.e. to the right of the reddened MS in the $J-H$ vs. $H-K$ colour-colour diagram. We selected 25 of the 181 sources as candidte YSOs, all which had good photometry (all errors below $0.1 \mathrm{mag}$ ). 20 of the 25 point sources are located towards the TMR (i.e. within the $I_{200}>140 \mathrm{MJy} \mathrm{sr}^{-1}$ region). The 5 objects which have the largest $H-K$ index of all the YSO candidate 2MASS sources are found as best candidates for highly reddened $\left(A_{\mathrm{V}}=15 \pm 5 \mathrm{mag}\right)$ young, low mass stars $\left(M<0.2 M_{\odot}\right)$ in TMC-1 CP (2MASS TMRS 23), in HCL2-B (2MASS TMRS 3), in HCL2-E (2MASS TMRS 15 and 10) at high $I_{200}$ regions. The 181 sources with CTT-like 

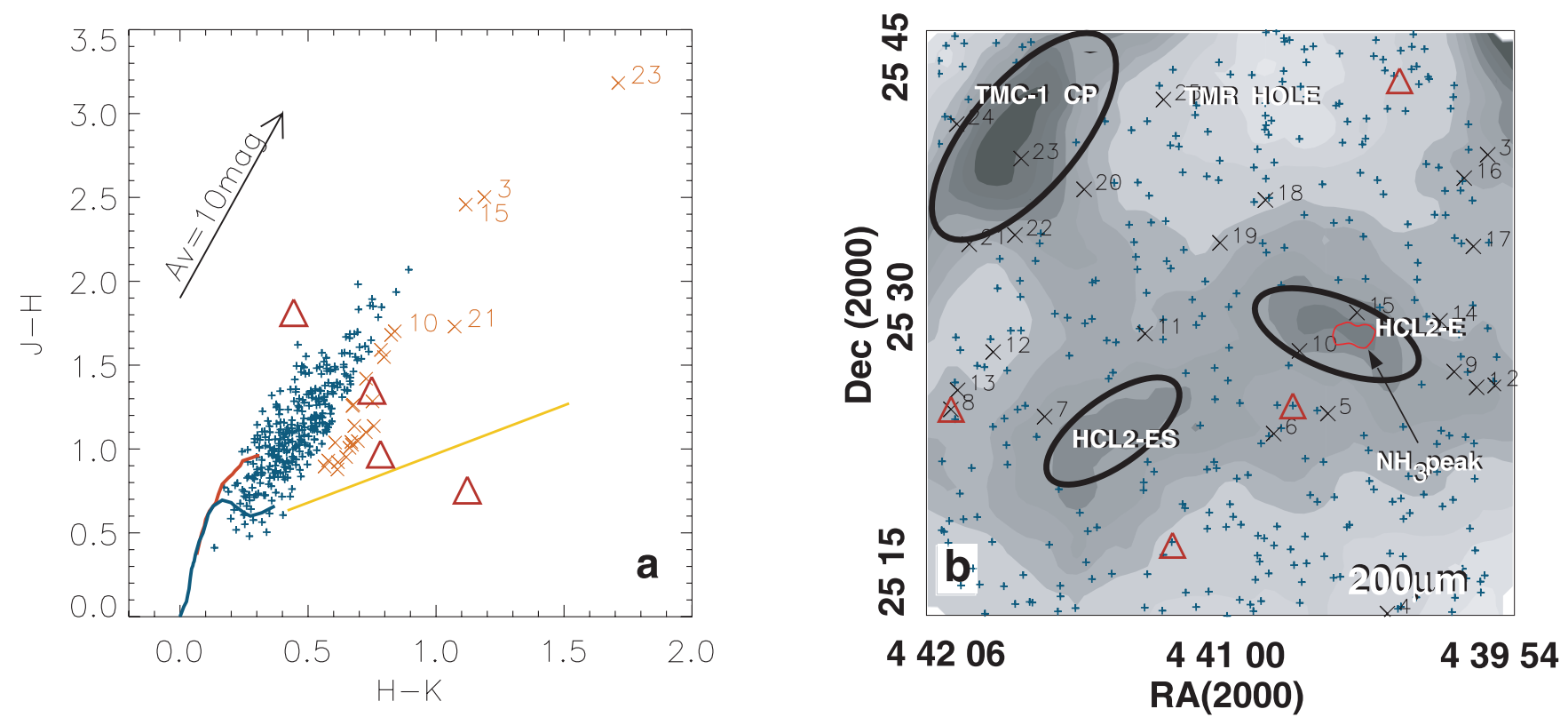

Fig. 6. a) $H-K_{\mathrm{S}}$ vs. $J-H$ colours of $2 \mathrm{MASS}$ sources of the region. Point sources falling inside the the locus of classical T Tauri stars (CTT, Meyer et al. 1997) and having a good photometry are marked with red $\times$-es. The reddened MS and evolved stars (with good photometry) are marked with small blue pluses. Four extended 2MASS objects are marked with red triangles. b) Grayscale map of the ISOPHOT $200 \mu \mathrm{m}$ surface brightness with 2MASS sources overlaid. The 25 candidate classical T Tauris (CTTs) (marked with black $\times$-es) are located around the density peaks of the cores. The reddened MS and evolved stars (with good photometry) are marked with small blue pluses. (This figure in available in colour in the electronic version.)

colours account for $23 \%$, the 25 YSO candidates for $3 \%$ of all 2MASS point sources towards the southern TMR. We note that the same regions of the NIR colour-colour diagram are populated with some evolutionary old objects (AGNs, PNs). To estimate the fraction of misclassified objects, the classification was repeated in a similar size reference region at $\approx 3^{\circ}$ distance from TMC-1. Only $1.3 \%$ of the 2MASS point sources of the reference field have CTT-like colours and none has an unreddened $H-K$ index over 0.9 . Further details on the nature of the 2MASS point sources of TMR and their relation to the cold and dense cores are given by Tóth et al. (2004).

Four extended 2MASS objects appear in the southern TMR, two of them with T Tauri-like colours (see triangles in Fig. 6). Their average total elliptical radius is $r_{\mathrm{ext}}=12.6^{\prime \prime}$ which corresponds to $8.6 \times 10^{-3} \mathrm{pc}$ at the distance of Taurus.

Itoh et al. (1996 and 1999, ITG) carried out a nearinfrared survey of the central 1 square degree region of HCL2. The limiting $K$ magnitude of their survey was $13.4 \mathrm{mag}$, and they detected 831 sources. Based on their colour-colour diagram, 50 sources were classified as YSO candidates. Recently Itoh et al. (2002) found that according to NIR spectra, 10 of their former YSO candidate sources were field stars, but approved 12 of those as YSOs. From the 15 ITG sources seen towards TMR south 5 are field stars and 1 source, ITG 40 is a $<0.2 M_{\odot}$ YSO with an age of $10^{6}$ to $10^{7}$ years. We listed the ITG YSO candidates in Table 4 with their 2MASS identifications. The confirmed field stars were left out. Murphy \& Myers (2003) detected 11 reddened NIR point sources in the $H$ and/or $K_{\mathrm{s}}$ bands towards TMC-1 CPa and considered none of those as YSO; however, for about half of them it can not be excluded.
YSO candidates located by X-ray measurements: Three ROSAT point sources appear towards the southern part of TMR with YSO-like hardness ratios. The first of them, HCL2 SE-2, is a very weak X-ray source, detected with $S / N>6$ in the central $30^{\prime}$ of the detector field of view, which ensures the quality (Briceno et al. 1999). It has no counterpart on any of the DSS2 blue, red, infrared plates. However, it is associated with a NIR nebulosity 2MASSX J04404265+2525466 with multiple peaks and classical T Tauri type NIR colours and a loose group of 5 reddened point sources within 2 ' search radius. HCL2 SE-2 is associated with HCL2-E and may be a very young object obscured by TMR. The 25 , and $60 \mu \mathrm{m}$ IRAS images have a slight excess at the position of HCL2 SE-2 and we found the IRAS point source IRAS $04378+2521$ within 1' distance.

Both the other two X-ray point sources were found in between HCL2-E and HCL2-B in TMR. The ROSAT source 1RXS J044001.3+253533 corresponds to TYC 1834-179-1 at a distance of $108 \mathrm{pc}$ and with the error bars of the Taurus cloud distances can be considered as a loose member.

Table 4 indicates that the dense and cold clouds of TMR are associated with candidate YSOs. Figure 8 presents the YSO distribution around the very cold core of HCL2-E. With the present dataset we cannot exclude the possibility of pure projection. Nevertheless it is likely that some of the faint point sources were formed in the clouds of the southern part of TMR.

\section{Discussion}

In this section we discuss the physical state of the clouds of TMR and the cores inside the clouds. Various possibilities are considered on the origin of TMR in view of the multiwavelength data. 
Table 4. Candidate young stellar objects of the region: reddened NIR point sources and nebulosities with T Tauri-like NIR colours, X-ray point sources. The classification, as indicated, is based on $J, H, K$ photometry, NIR spectroscopy, or X-ray hardness ratio (HR). The columns are: (1) object name, (2-3) equatorial coordinates, (4) classification method, (5) associated cloud and separation, (6) other identifications of the same source.

\begin{tabular}{|c|c|c|c|c|c|c|}
\hline Source & RA (2000) & $\operatorname{Dec}(2000)$ & Class & Cloud & Sep. & Other identifications \\
\hline 2MASS TMRS1 & $04^{\mathrm{h}} 39^{\mathrm{m}} 59^{\mathrm{s}} .3$ & $+25^{\circ} 26^{\prime} 43^{\prime \prime}$ & JHK & & & \\
\hline 2MASS TMRS2 & $\begin{array}{lll}04 & 39 & 54.9\end{array}$ & +252649 & $J H K$ & & & \\
\hline 2MASS TMRS3 & $\begin{array}{lll}04 & 39 & 56.7\end{array}$ & +253836 & $J H K$ & HCL2-B & $1^{\prime}$ & \\
\hline 2MASS TMRS4 & $\begin{array}{lll}04 & 40 & 20.3\end{array}$ & +251510 & $J H K$ & & & \\
\hline 2MASS TMRS5 & $\begin{array}{lll}04 & 40 & 34.4\end{array}$ & +252523 & JHK & & & \\
\hline 2MASS TMRS6 & $\begin{array}{lll}04 & 40 & 47.2\end{array}$ & +252421 & $J H K$ & & & \\
\hline 2MASS TMRS7 & $\begin{array}{lll}04 & 41 & 41.3\end{array}$ & +252514 & $J H K$ & HCL2-ES & $2^{\prime}$ & \\
\hline 2MASS TMRS8 & $\begin{array}{lll}04 & 42 & 03.4\end{array}$ & +252536 & $J H K$ & & & \\
\hline 2MASS TMRS9 & $\begin{array}{lll}04 & 40 & 04.6\end{array}$ & +252731 & $J H K$ & & & \\
\hline 2MASS TMRS10 & $\begin{array}{lll}04 & 40 & 41.1\end{array}$ & +252835 & $J H K$ & HCL2-E & $0^{\prime}$ & IRAS $04378+2521$ (within err. ellipse) \\
\hline 2MASS TMRS11 & $\begin{array}{lll}04 & 41 & 17.6\end{array}$ & +252928 & JHK & & & \\
\hline 2MASS TMRS12 & $\begin{array}{lll}04 & 41 \quad 53.4\end{array}$ & +252832 & JHK & & & \\
\hline 2MASS TMRS13 & $\begin{array}{lll}04 & 42 & 01.8\end{array}$ & +252635 & JHK & & & \\
\hline 2MASS TMRS14 & $\begin{array}{lll}04 & 40 & 07.9\end{array}$ & +253007 & JHK & HCL2-E & $2^{\prime}$ & \\
\hline 2MASS TMRS15 & $\begin{array}{lll}04 & 40 & 27.5\end{array}$ & +253033 & $J H K$ & HCL2-E & $0^{\prime}$ & \\
\hline 2MASS TMRS16 & $\begin{array}{lll}04 & 40 & 02.2\end{array}$ & +253725 & JHK & HCL2-B & $3^{\prime}$ & \\
\hline 2MASS TMRS17 & $\begin{array}{lll}04 & 40 & 00.0\end{array}$ & +253356 & $J H K$ & & & \\
\hline 2MASS TMRS18 & $\begin{array}{lll}04 & 40 & 49.1\end{array}$ & +253618 & JHK & & & \\
\hline 2MASS TMRS19 & $\begin{array}{lll}04 & 40 & 59.9\end{array}$ & +253406 & $J H K$ & & & \\
\hline 2MASS TMRS20 & $\begin{array}{lll}04 & 41 & 31.9\end{array}$ & +253650 & JHK & TMC-1 CP & $1^{\prime}$ & \\
\hline 2MASS TMRS21 & $\begin{array}{lll}04 & 41 \quad 59.0\end{array}$ & +253402 & $J H K$ & TMC-1 CP & $0^{\prime}$ & \\
\hline 2MASS TMRS22 & $\begin{array}{lll}04 & 41 & 48.3\end{array}$ & +253431 & $J H K$ & TMC-1 CP & $0^{\prime}$ & \\
\hline 2MASS TMRS23 & $\begin{array}{lll}04 & 41 & 46.8\end{array}$ & +253825 & $J H K$ & TMC-1 CP & $0^{\prime}$ & \\
\hline 2MASS TMRS24 & $\begin{array}{lll}04 & 42 & 02.0\end{array}$ & +254011 & JHK & TMC-1 CP & $0^{\prime}$ & \\
\hline 2MASS TMRS25 & $\begin{array}{lll}04 & 41 & 13.2\end{array}$ & +254125 & $J H K$ & TMC-1 CP & $3^{\prime}$ & \\
\hline ITG 26 & $04^{\mathrm{h}} 40^{\mathrm{m}} 28^{\mathrm{s}} 3$ & $+25^{\circ} 26^{\prime} 56^{\prime \prime}$ & $\overline{J H K}$ & HCL2-E & $\overline{4^{\prime}}$ & 2MASS J043959.39+253120.8 \\
\hline ITG 31 & $\begin{array}{lll}04 & 41 & 02.7\end{array}$ & +253948 & $J H K$ & TMC-1 CP & $6^{\prime}$ & 2MASS J043922.60+253428.2 \\
\hline ITG 32 & $\begin{array}{lll}04 & 41 & 04.0\end{array}$ & +253023 & $J H K$ & HCL2-ES & $2^{\prime}$ & 2MASS J043940.80+253544.6 \\
\hline ITG 37 & $\begin{array}{lll}04 & 41 & 17.9\end{array}$ & +254208 & $J H K$ & TMC-1 CP & $3^{\prime}$ & 2MASS J043912.01+254110.1 \\
\hline ITG 38 & $\begin{array}{lll}04 & 41 & 19.2\end{array}$ & +252426 & $J H K$ & HCL2-ES & $2^{\prime}$ & 2MASS J043922.51+254032.4 \\
\hline ITG 40 & $\begin{array}{lll}04 & 41 \quad 24.7\end{array}$ & +254353 & NIRspec. & TMC-1 CP & $0^{\prime}$ & 2MASS J043956.66+253836.3 \\
\hline ITG 44 & $\begin{array}{lll}04 & 41 & 43.3\end{array}$ & +252411 & $J H K$ & TMC- $1 \mathrm{NH}_{3}$ & $3^{\prime}$ & 2MASS J043944.58+254308.3 \\
\hline ITG 47 & $\begin{array}{lll}04 & 42 & 00.0\end{array}$ & +252250 & $J H K$ & TMC- $1 \mathrm{NH}_{3}$ & $3^{\prime}$ & 2MASS J043840.03+254518.6 \\
\hline ITG 48 & $\begin{array}{lll}04 & 42 & 05.0\end{array}$ & $+25 \quad 2257$ & $J H K$ & TMC- $1 \mathrm{NH}_{3}$ & $3^{\prime}$ & 2MASS J043835.16+254825.6 \\
\hline ITG 49 & $\begin{array}{lll}04 & 42 & 06.0\end{array}$ & +253911 & JHK & TMC- $1 \mathrm{NH}_{3}$ & $3^{\prime}$ & 2MASS J043920.91+254502.1 \\
\hline HCL2 SE-2 & $04^{\mathrm{h}} 40^{\mathrm{m}} 42^{\mathrm{s}} .6$ & $+25^{\circ} 26^{\prime} 21^{\prime \prime}$ & HR, FIR, JHK & HCL2-E & $3^{\prime}$ & IRAS 04378+2521, 2MASSX J04404265+2525466 \\
\hline 1RXS J044001.3+253408 & $\begin{array}{lll}04 & 40 & 01.3\end{array}$ & +253408 & $\mathrm{HR}$ & HCL2-E & $3^{\prime}$ & \\
\hline 1RXS J044009.8+253533 & $\begin{array}{lll}04 & 40 & 09.8\end{array}$ & $+25 \quad 35 \quad 33$ & HR & HCL2-E & $4^{\prime}$ & \\
\hline
\end{tabular}

\subsection{Gas and dust in $T M R$}

The southern part of TMR is a proper region for gas-dust comparative studies, since it lacks luminous FIR point sources. While Fig. 7a shows a general correlation, the regions have slightly different 60 to $100 \mu \mathrm{m}$ colours. The difference between TMC-1 CP and the rest of TMR south is remarkable in Fig. 7b, i.e. TMC-1 CP has a clear excess in $200 \mu \mathrm{m}$ surface brightness (or deficit in $100 \mu \mathrm{m}$ ). To test the relation of gas column density and dust colour the $\mathrm{C}^{18} \mathrm{O}$ intensity is plotted with the $\Delta I_{200} / \Delta I_{100}$ ratio in Fig. $7 \mathrm{~d}$. TMC-1 is again well off the trend, further emphasizing the FIR colour difference between TMC-1 CP and the rest of TMR south.
Figure $7 \mathrm{c}$ indicates a generally valid linear correlation between extinction and FIR surface brightness with the exception of the $I_{200}$ brightest regions. i.e. the relation is not valid at some positions in HCL-2E and towards the densest cold cloud, TMC-1 CP. Figure 6a shows a rough classification of the 2MASS point sources of TMR south. The Padoan et al. (2002) 2MASS-based extinction values are strongly contaminated by the associated $\mathrm{T}$ Tauri type stars in the areas of highest extinction, since there appear to be practically no background field stars in the densest cores in Fig. 6b. Accordingly the extinction of TMC-1 CP is underestimated, and the corresponding points may actualy fall near the fitted line in Fig. 7c. Since 

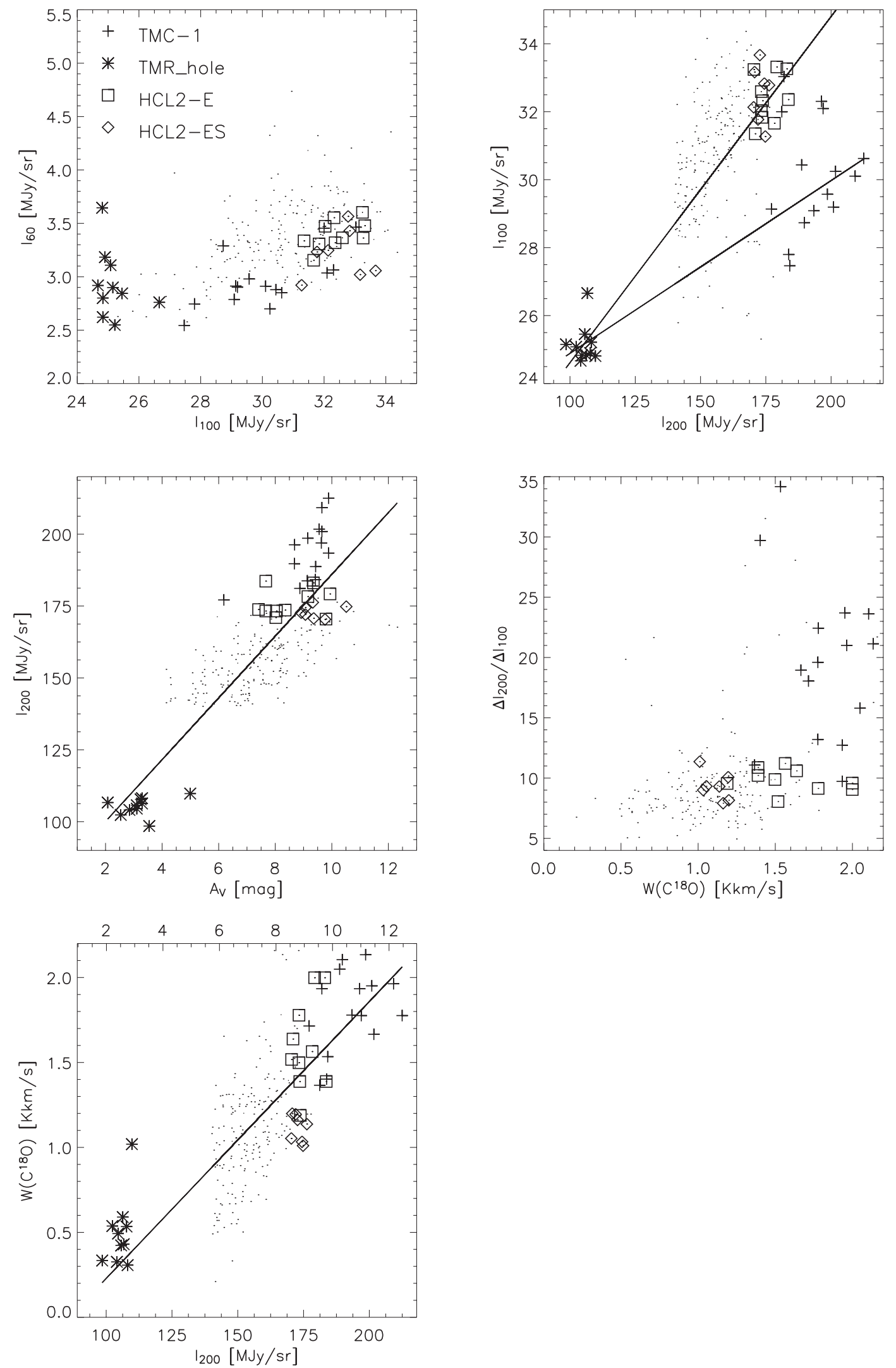

Fig. 7. a) $I_{60}$ vs. $I_{100}$ for all pixels of TMR (small dots). Different symbols were overlaid for the different parts of TMR and the hole, as indicated in the upper left corner. b) $I_{200}$ vs. $I_{100}$ with representative slopes fitted to the data points of TMC-1 and HCL2-E. c) $I_{200}$ vs. $A_{\mathrm{V}}$ with line fitted to all points. TMC-1 shows $I_{200}$ excess d) $W\left(\mathrm{C}^{18} \mathrm{O}\right)$ vs. $\Delta I_{200} / \Delta I_{100}$, the high ratios occur only inside the molecular cloud. e) $W\left(\mathrm{C}^{18} \mathrm{O}\right)$ vs. $I_{200}$ correlation in TMR. The correlation coefficient is $73 \%$. 
Table 5. Parameters of cloud cores inside the cold ISOPHOT clouds TMC-1 CP and HCL2-E. References for other names: (1) Onishi et al. (2002), (2) Hirahara et al. (1992). (3) Lee et al. (2001), (4) Langer et al. (1995).

\begin{tabular}{|c|c|c|c|c|c|c|c|c|c|c|}
\hline $\begin{array}{l}\text { Dense } \\
\text { core }\end{array}$ & $\mathrm{RA}(2000)$ & $\operatorname{Dec}(2000)$ & $\begin{array}{c}d \\
{[\mathrm{pc}]}\end{array}$ & $a / b$ & $\begin{array}{c}\Delta v \\
\mathrm{~km} \mathrm{~s}^{-1}\end{array}$ & $\begin{array}{c}v_{\mathrm{LSR}} \\
\mathrm{km} \mathrm{s}^{-1} \\
\end{array}$ & $\begin{array}{c}M \\
M_{\odot}\end{array}$ & $\begin{array}{c}n\left(\mathrm{H}_{2}\right) \\
10^{5} \mathrm{~cm}^{-3}\end{array}$ & $\begin{array}{c}M_{\text {vir }} \\
M_{\odot}\end{array}$ & Other name, ref. \\
\hline HCL2-Eab & $04^{\mathrm{h}} 40^{\mathrm{m}} 31^{\mathrm{s}} .0$ & $+25^{\circ} 29^{\prime} 27^{\prime \prime}$ & 0.12 & 3.0 & 0.7 & 4.7 & 7.9 & 2.6 & 6.2 & \\
\hline HCL2-Ec & $\begin{array}{lll}04 & 40 & 41.6\end{array}$ & +253026 & 0.08 & 2.8 & 0.8 & 5.0 & 2.0 & 1.3 & 5.4 & Onishi42c (1) \\
\hline TMC-1 CPa & $\begin{array}{lll}04 & 41 & 34.0\end{array}$ & +254451 & 0.15 & 3.0 & 0.8 & 5.7 & 8.0 & 2.4 & 8.1 & HiraharaC (2), TMC1 (3) \\
\hline TMC-1 CPb & $\begin{array}{lll}04 & 41 & 45.2\end{array}$ & +254027 & 0.18 & 1.5 & 0.7 & 5.8 & 12.9 & 4.1 & 9.2 & HiraharaD (2), Core D(4) \\
\hline TMC-1 CPc & 0441 & +253626 & 0.06 & 1.0 & 0.7 & 5.6 & 1.2 & 1.8 & 3.1 & HiraharaE (2) \\
\hline
\end{tabular}

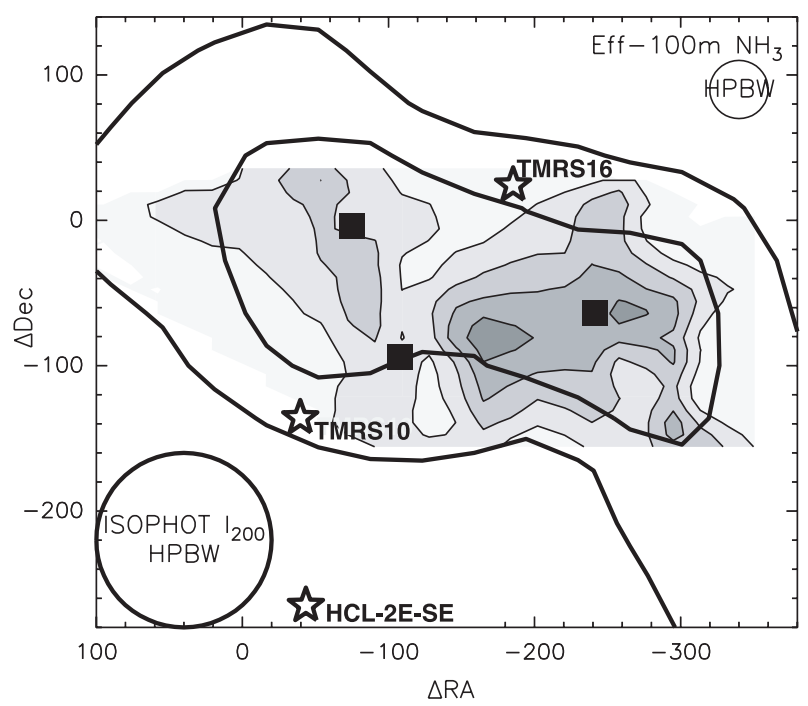

Fig. 8. Grayscale map of $\mathrm{NH}_{3}(1,1)$ line area distribution at the $I_{200}$ peak in the HCL2-E cloud. The origin is at: RA(1950) = $4^{\mathrm{h}} 37^{\mathrm{m}} 42^{\mathrm{s}}, \operatorname{Dec}(1950)=25^{\circ} 25^{\prime}$. The continuous contours are drawn from $0.4 \mathrm{~K} \mathrm{~km} \mathrm{~s}^{-1}\left(3 \sigma \mathrm{NH}_{3}(1,1)\right.$ line area) by $0.2 \mathrm{~K} \mathrm{~km} \mathrm{~s}^{-1}$. The beam-width $\left(H P B W=40^{\prime \prime}\right)$ of the Effelsberg-100 $\mathrm{m}$ telescope is shown in the upper right corner. The ISOPHOT $200 \mu \mathrm{m}$ surface brightness contours of 165 and $175 \mathrm{MJy} \mathrm{sr}^{-1}$ are drawn with dashed lines indicating the location of the FIR peak in HCL2-E. The HPBW of ISOPHOT C200 is shown in the lower left corner. The central positions of the $\mathrm{H}^{13} \mathrm{CO}^{+}$cores of 42a,b,c of Onishi (2002) are marked with black squares. The HCL2 SE-2 X-ray/IRAS pointsource and two more YSO candidates are marked with star and annotation.

the dust colour temperature is definitely lower in TMC-1 CP than elsewhere in TMR south, a deficiency should be seen in the FIR surface brightness (dominated by $I_{200}$ ), while a linear correlation was observed between $W\left(\mathrm{C}^{18} \mathrm{O}\right)$ and $I_{200}$ as shown in Fig. 7e. There the relation found between $A_{\mathrm{V}}$ and $I_{200}$ (see Fig. 7c) is used to print the approximated $A_{\mathrm{V}}$ values on the upper axis. The reason that there is no relative drop in $I_{200}$ can be an increased FIR emissivity of dust in the densest regions. A similar effect has been found recently by del Burgo et al. (2003) in dark clouds with moderate extinction (1 mag < $\left.A_{\mathrm{V}}<5 \mathrm{mag}\right)$. Such a variation was claimed to indicate the presence of large fluffy dust particles, a dust population so far found in moderately dense regions like an $\left(A_{\mathrm{V}} \leq 4 \mathrm{mag}\right)$ clump in a filament in Taurus (Stepnik et al. 2003). In the presented case of TMR the effect is shown in the $A_{\mathrm{V}}>4$ mag regime.

As Abergel et al. (1996) pointed out, in general the cold component of the $100 \mu \mathrm{m}$ radiation is well correlated with the
${ }^{13} \mathrm{CO}(1-0)$ velocity integrated emission in Taurus. The apparent ${ }^{13} \mathrm{CO}$ distribution however looks smooth, only the large structures are seen. The $\mathrm{C}^{18} \mathrm{O}$ distribution pinpoints the density enhancement regions. As our Fig. 3 shows, the $\mathrm{C}^{18} \mathrm{O}$ intensity distributioncompares also much better to the distribution of $200 \mu \mathrm{m}$ surface brightness than to any of the IRAS images. The linear correlation coefficient of $W\left(\mathrm{C}^{18} \mathrm{O}\right)$ vs. $I_{200}$ was found to be $73 \%$ (see Fig. 7e). The correlation coefficient, when $I_{200}$ is compared to the blue or the red shifted (i.e. $v<6 \mathrm{~km} \mathrm{~s}^{-1}$ or $v>6 \mathrm{~km} \mathrm{~s}^{-1}$ ) part of the integrated line area is below $60 \%$. We note that the correlation coefficient of ${ }^{13} \mathrm{CO}$ vs. $I_{100}$ was $57 \%$ in Taurus (Abergel et al. 1994). The correlation of the ISOPHOT FIR surface brightness with the $\mathrm{C}^{18} \mathrm{O}$ integrated intensity is shown in Fig. 7e.

We have found $T_{\mathrm{d}} \approx T_{\text {kin }}$ in HCL-2E and in TMC-1 CP which is expected for clouds with $n\left(\mathrm{H}_{2}\right)>10^{5} \mathrm{~cm}^{-3}$ densities. We note that the agreement is excellent when the $100 \mu \mathrm{m}$ intensity is corrected for the warm dust contribution. The combination of very low dust temperatures and high column densities may be the reason for the unique richness of TMC-1 CP in complex molecules. The molecules are released from the dust grain surfaces in a rate depending on the excitation of the grains. Consequently the molecules are stuck longer on the grains in TMC-1 CP than in other dark clouds. When large dust particles with (organic) mantles dominate the dust particle distribution, the rich chemistry in TMC-1 is achieved through a mantle removal mechanism. An explosive desorption of photolyzed ices can be induced by MHD waves as they propagate within the cloud and the mantle molecules are ejected to the gas phase as proposed by Markwick et al. (2000).

\subsection{Cold clouds of the southern TMR}

Other molecular line observations: The Nobeyama-45 m $\mathrm{H}^{13} \mathrm{CO}^{+}(1-0)$ survey by Onishi et al. (2002, OMK) covered all the $\mathrm{C}^{18} \mathrm{O}$ bright $\left(T\left(\mathrm{C}^{18} \mathrm{O}\right) \geq 1.4 \mathrm{~K} \mathrm{~km} \mathrm{~s}^{-1}\right)$ Taurus clouds, and located 7 dense cores in TMR. Exploring the substructure of the clouds of TMR south we crosschecked $\mathrm{H}^{13} \mathrm{CO}^{+}$channel maps with similarly high resolution observations of Langer et al. (1995), Olano et al. (1988) and Takakuwa et al. (2000). Physical parameters of the dense cores located are listed in Table 5, where the columns are: (1) core name, (2-3) J2000 equatorial coordinates of geometrical centre, (4) diameter, (5) axial ratio (6) $\mathrm{H}^{13} \mathrm{CO}^{+}$line width, (7) $\mathrm{H}^{13} \mathrm{CO}^{+}$velocity, (8) total gas mass (9) $\mathrm{H}_{2}$ density (10) estimated virial mass (11) other names for the core. 
TMC-1 The most massive dense $\mathrm{H}^{13} \mathrm{CO}^{+}$clump of the whole Taurus complex is OMK 43 corresponding to TMC-1. The dense part of TMC- 1 extends from $l=174^{\circ} .1$ to $l=174^{\circ} .48$ along $b=-13.56$ with a width of $4^{\prime} \pm 1^{\prime}$, as outlined by the $W\left(\mathrm{H}^{13} \mathrm{CO}^{+}\right)=0.25 \mathrm{~K} \mathrm{~km} \mathrm{~s}^{-1}$ contour of the $\mathrm{H}^{13} \mathrm{CO}$ line intensity. Its largest core is double peaked at TMC-1(NH3) the $\mathrm{NH}_{3}$ maximum region of TMC-1, NE of IRAS $04381+2540$, few arcminutes outside of our ISOPHOT image. Further local maxima of $W\left(\mathrm{H}^{13} \mathrm{CO}^{+}\right)$are seen in TMC-1 CP.

TMC-1 CP: The column density of the $\mathrm{OH}$ radical in TMC-1 also reaches its maximum at TMC-1 CP (Harju et al. 2000). We note that this coincides well with the $I_{200} \geq 170 \mathrm{MJy} \mathrm{sr}^{-1} \mathrm{re}-$ gion. i.e. TMC-1 CP, the SE half of the TMC-1 ridge is not only the region of maximum line intensity of carbon chain molecules and $\mathrm{OH}$, but also a strong $200 \mu \mathrm{m}$ source. The cloud is on the other hand practically free of $60 \mu \mathrm{m}$ emission and faint in $100 \mu \mathrm{m}$; thus it is a location of very cold dust with $\approx 11 \mathrm{~K}$ dust temperature. The dense gas as traced by $\mathrm{NH}_{3}$ is also very cold with $T_{\text {kin }} \approx 9 \mathrm{~K}$.

Fragments in TMC-1CP: are seen down to $0.01 \mathrm{pc}$ scale as shown by interferometric measurements of Langer et al. (1995). We locate substructures with sizes $\gtrsim 0.05 \mathrm{pc}\left(80^{\prime \prime}\right)$. The cores TMC-1 CP a,b,c lie along the cyanopolyyne peak region of TMC-1, with TMC-1 CPa falling at the northern boundary of our ISOPHOT image.

TMC-1 CP-a: This core appears $4^{\prime}$ south of TMC-1(NH3), at the northern border of the ISOPHOT image. Both our $\mathrm{H}^{13} \mathrm{CO}^{+}$data and the $\mathrm{C}^{18} \mathrm{O}(1-0)$ data by Langer et al. (1995) show a $2^{\prime} \times 1^{\prime}$ local maximum with integrated intensities of $\approx 0.9 \mathrm{~K} \mathrm{~km} \mathrm{~s}^{-1}$ and $>2 \mathrm{~K} \mathrm{~km} \mathrm{~s}^{-1}$ respectively. This is also a $\mathrm{HC}_{7} \mathrm{~N}$ local maximum of Olano et al. (1988) with $T\left(\mathrm{HC}_{7} \mathrm{~N}(J=\right.$ 21-20)) > $1 \mathrm{~K} \mathrm{~W}$. Lee et al. (2001) listed this source as TMC1 $\left(\operatorname{RA}(1950)=04^{\mathrm{h}} 38^{\mathrm{m}} 29^{\mathrm{s}} \operatorname{Dec}(1950)=+25^{\circ} 39^{\prime} 01^{\prime \prime}\right)$ and derived $0.60 \times 10^{5} M_{\odot} \mathrm{yr}^{-1}$ infall rate. Murphy \& Myers (2003) measured a very high reddening $E(B-V)=46.2$ towards TMC-1 CPa and derived density and mass lower limits of $1.6 \times$ $10^{5} \mathrm{~cm}^{-3}$ and $2.2 M_{\odot}$ respectively. Harvey et al. (2002) detected 3 point sources towards TMC-1 CPa in the $3.6 \mathrm{~cm}$ continuum with the VLA (in D configuration). Two of their sources have 2MASS counterparts, both with YSO-like NIR colours but with magnitude errors larger than 0.1 mag. TMC- 1 CP-a is a gravitationally bound core with detected infall.

TMC-1 CP-b was first isolated in column density maps by Hirahara et al. (1992) who named it as "core D" it coincides with the $\mathrm{HC}_{7} \mathrm{~N}(J=21-20)$ peak of TMC-1 with $T\left(\mathrm{HC}_{7} \mathrm{~N}(J=21-20)\right)>2 \mathrm{~K}$ (Olano et al. 1988 , see their Fig. 2). It appears as a $W\left(\mathrm{H}^{13} \mathrm{CO}^{+}\right)>0.8 \mathrm{~K} \mathrm{~km} \mathrm{~s}^{-1}$ local maximum in the Onishi et al. (2002) maps. TMC-1 CP-b was also identified in $\mathrm{C}^{18} \mathrm{O}(1-0)$ by Langer et al. (1995) who estimated a mass of $2.2 M_{\odot}$ already enough to gravitationally bind the core. We found that TMC-1 CP-b can be two times more massive. The Langer et al. (1995) $\operatorname{CCS}\left(2_{1}-1_{0}\right)$ map resolved 3 (as they estimated) unbound clumps inside TMC-1 CP-b. There is one YSO candidate seen projected onto it and of further 2 are associated.
TMC-1 CP-c: According to Olano et al. (1988, see their Fig. 1) there is a more or less separated, small $\left(d \approx 80^{\prime \prime}\right)$ $T_{\text {main }}\left(\mathrm{NH}_{3}(1,1)\right)>2 \mathrm{~K}$ local maximumum at the $\mathrm{SE}$ end of the elongated TMC-1. It coincides with a $T_{\mathrm{b}}\left(\mathrm{C}^{18} \mathrm{O}(1-0)\right)>2 \mathrm{~K}$ peak (Langer et al. 1995, see their Fig. 1a). The column density is $N\left(\mathrm{H}_{2}\right) \approx 1.5 \times 10^{21} \mathrm{~cm}^{-2}$ if their formula is applied with $\left.T_{\mathrm{ex}} \mathrm{C}^{18} \mathrm{O}(2-1)\right)=7 \mathrm{~K}$ and $\Delta v=0.4 \mathrm{~km} \mathrm{~s}^{-1}$ i.e. $N\left(\mathrm{H}_{2}\right)=9.9 \times$ $10^{20}\left[1-\exp \left(-5.3 / T_{\mathrm{ex}} \mathrm{C}^{18} \mathrm{O}(2-1)\right)\right]^{-1} \times \int T_{\mathrm{b}}\left(\mathrm{C}^{18} \mathrm{O}(2-1)\right) \mathrm{d} v$. The column density is similar $\left(N\left(\mathrm{H}_{2}\right) \approx 1.6 \times 10^{21} \mathrm{~cm}^{-2}\right)$ when calculated from $\mathrm{NH}_{3}(1,1)$ according to Olano et al. (1988): $N\left(\mathrm{H}_{2}\right) \approx 3.3 \times 10^{7} \times N\left(\mathrm{NH}_{3}\right)=1.4 \times 10^{13} Z\left(T_{\mathrm{kin}}\right) \tau \Delta v T_{\mathrm{ex}}$, where $Z\left(T_{\text {kin }}\right)=4.4$ when $T_{\text {kin }}=10 \mathrm{~K}, \Delta v=0.7 \mathrm{~km} \mathrm{~s}^{-1}$ and $\tau \approx 0.5$ as we measured nearby. With a total gas mass of $1.2 M_{\odot}$ this is an unbound clump, since the virial mass would be around $3 M_{\odot}$. Still there are 2 YSO candidates associated.

HCL2-E: is fainter by only $20 \%$ than TMC-1 CP in $\mathrm{C}^{18} \mathrm{O}(1-0)$ and by $10 \%$ in $200 \mu \mathrm{m}$ surface brightness. With $12.6 \mathrm{~K}$ dust temperature and to 10.3 to $14.7 \mathrm{~K}$ kinetic temperature HCL2-E is somewhat warmer than TMC-1CP. HCL2-E appears as bright as TMC-1 CP in the $1667 \mathrm{MHz}$ and $165 \mathrm{MHz}$ hyperfine lines of $\mathrm{OH}$ (see at the edge of Fig. 2 of Harju et al. 2000). HCL2-E hosts two molecular cloud cores inside the $I_{200} \geq$ $175 \mathrm{MJy} \mathrm{sr}^{-1}$ region, according to our $\mathrm{NH}_{3}$ measurements (see Fig. 8): the double peaked HCL2-Eab, and HCL2-Ec. These lie inside the $I_{200}=175 \mathrm{MJy} \mathrm{sr}^{-1}$ contour. A corresponding group of $3 \mathrm{H}^{13} \mathrm{CO}^{+}$cores OMK 42a,b,c with densities of $n\left(\mathrm{H}_{2}\right)=$ $(1.5 \pm 0.2) \times 10^{5} \mathrm{~cm}^{-3}$ was listed by Onishi et al. (2002). We note the agreement between the $\mathrm{NH}_{3}$ and the $\mathrm{H}^{13} \mathrm{CO}^{+}$ based densities. The velocities of the $\mathrm{H}^{13} \mathrm{CO}^{+}$cores agree with the $\mathrm{NH}_{3}$ velocities and both are significantly lower than the $\mathrm{C}^{18} \mathrm{O}$ velocities towards the same positions. Since $\mathrm{C}^{18} \mathrm{O}$ is becoming optically thick, it may trace only the envelope gas of the dense cores; this gas envelope apparently has a velocity offset being redshifted by $0.7 \pm 0.3 \mathrm{~km} \mathrm{~s}^{-1}$.

HCL2-Eab core is $3^{\prime} \times 2^{\prime}$ E-W elongated with a velocity gradient of $0.1 \mathrm{~km} \mathrm{~s}^{-1} / 1^{\prime}$ from ENE to WSW. A weak $\mathrm{NH}_{3}(2,2)$ line was detected after averaging spectra in a $1^{\prime}$ diameter region at HLC2-Ea, while it was 4 times stronger at the HLC2-Eb position (see Fig. 5). We interpret this to mean that the HLC2-Eab core also shows a gradient (increase from W to E) also in kinetic temperature, as seen from $T_{1,2}$ rotational temperature variations of $\mathrm{NH}_{3}$. HLC2-Ea is the cold part with $T_{\text {kin }} \lesssim 10 \mathrm{~K}$ and the SE side, facing the YSO HCL2 SE-2 is warmer with $T_{\text {kin }} \approx 15 \mathrm{~K}$. This, together with the velocity gradient may indicate an interaction with the FIR pointsource HCL2 SE-2 south of HCL2-E.

HCL2-Eab's double peaked structure has been found by $\mathrm{H}^{13} \mathrm{CO}^{+}$mapping and was catalogued as \#43a and \#43b cores by Onishi et al. (2002). While \#43a corresponds well to the eastern local maximum of $T\left(\mathrm{NH}_{3}(1,1)\right)$, \#43b is located $1^{\prime}$ east of HCL2-Eb (spectra are shown in Fig. 5) which corresponds to the $\tau_{\text {main }}\left(\mathrm{NH}_{3}(1,1)\right)$ maximum position in HLC2-E.

HCL2-Eab is a gravitationally bound core and has an asociated T Tauri like pointsource HCL2 SE-2, at $<3^{\prime}$ distance, a 
YSO candidate, 2MASS TMR15, seen at its northern boundary, 2MASS TMR10 (associated with IRAS 04378+2521) lies within $3^{\prime}$ and another one, ITG 26, at $4^{\prime}$ distance.

The HLC2-Ec core is $2^{\prime} \times 0.6^{\prime} \mathrm{N}-\mathrm{S}$ elongated We consider this as a separate core since its velocity $v_{\mathrm{LSR}}=5.1 \mathrm{~km} \mathrm{~s}^{-1}$ is $0.4 \mathrm{~km} \mathrm{~s}^{-1}$ different from that of HCL2-Eab, it has a different orientation and is separate both in the $\mathrm{NH}_{3}(1,1)$ and $\mathrm{H}^{13} \mathrm{CO}^{+}$lines. It corresponds well to core \#43c by Onishi et al. (2002). It is gravitationally unbound.

HCL2-ES is very cold with $\approx 13 \mathrm{~K}$ dust temperature, but less structured than TMC-1CP or HCL2-E. Visual starcount-based extinction maps and ${ }^{13} \mathrm{CO}(1-0)$ maps show HCL2-E/HCL2-ES as one cloud; as Heyer (1988) stated: the second most massive of the whole Taurus with $31.7 M_{\odot}$. $\mathrm{H}^{13} \mathrm{CO}^{+}$was not detected in HCL2-ES with $1^{\prime}$ grid spacing $(3 \times$ under-sampled). Both the temperature and the level of turbulence are low as seen from the narrow $\mathrm{C}^{18} \mathrm{O}$ lines. It is however at a $T\left(\mathrm{C}^{18} \mathrm{O}\right)>2 \mathrm{~K}$ maximum of the southern TMR, and a local maximum of $A_{\mathrm{V}}$. HCL2-ES is without any well defined cloud core. We assume it is evolutionary the youngest sub-cloud in TMR; still there is one YSO candidate, 2MASS TMRS 7, seen associated.

\subsection{Virial stability and star formation}

Stability of the very cold clouds and cloud cores: The thermo dynamical virial mass $M_{\mathrm{vir}} /\left[M_{\odot}\right]$ of a homogenous, spherical cloud can be estimated from the radius $R /[\mathrm{pc}]$, and velocity dispersion $\delta v /\left[\mathrm{km} \mathrm{s}^{-1}\right]$ as $M_{\mathrm{vir}}=210 R \delta v^{2}$. Accordingly TMC-1 CP and HCL2-E are gravitationally bound, HCL2-ES is not. Three of the five dense cores in Table 5 are gravitationally bound. We consider these very cold cores of TMR as preprotostellar cores in thermo-dynamical instability. If this is the case magnetic braking does not play a major role in supporting the cloud cores, it only slows down the collapse. Since various authors estimate the clouds of HCL2 to be older than a few free fall times the magnetic pressure of the cores might have already been decreased by ambipolar diffusion. The associated YSO-like NIR point sources may trace an early phase in the evolution of the cloud as they are seen are all over TMR and not only at the ISM density peaks.

HI gas associated with HCL2: A large HI cloud is apparent at HCL2 in the HI $21 \mathrm{~cm}$ data of the Leiden/Dwingeloo HI survey (Hartmann \& Burton 1999). The $4.8 \times 2.4 \mathrm{pc}$ size $\mathrm{HI}$ cloud is associated with TMR at $3.1 \mathrm{~km} \mathrm{~s}^{-1}<v_{\mathrm{LSR}}<$ $7.2 \mathrm{~km} \mathrm{~s}^{-1}$ velocities. The total HI column density is $N(\mathrm{HI}) \approx$ $4.4 \times 10^{20} \mathrm{~cm}^{-2}$ towards the HCL2 cloud. We compare the atomic and molecular gas distributions in Fig. 9. The HI line area contours of $341 \mathrm{~K} \mathrm{~km} \mathrm{~s}^{-1}$ and $352 \mathrm{~K} \mathrm{~km} \mathrm{~s}^{-1}$ (red dashed line) are overlaid on the gray scale image of the Nagoya- $4 \mathrm{~m}$ $W\left(\mathrm{C}^{18} \mathrm{O}\right)$ distribution. $\mathrm{A} \approx 10 \mathrm{~K} \mathrm{~km} \mathrm{~s}^{-1}$ average excess $21 \mathrm{~cm}$ line intensity corresponds to $N(\mathrm{HI}) \approx 1.9 \times 10^{19} \mathrm{~cm}^{-2} \mathrm{HI}$ column density and a total $\mathrm{HI}$ mass of $17 M_{\odot}$. The HI $21 \mathrm{~cm}$ spectra were found by Wilson \& Mink (1977) to be selfabsorbed towards HCL2 indicating that HCL2 is associated also with cold $\left(T_{\text {kin }}<30 \mathrm{~K}\right) \mathrm{HI}$ gas. The cold HI distribution

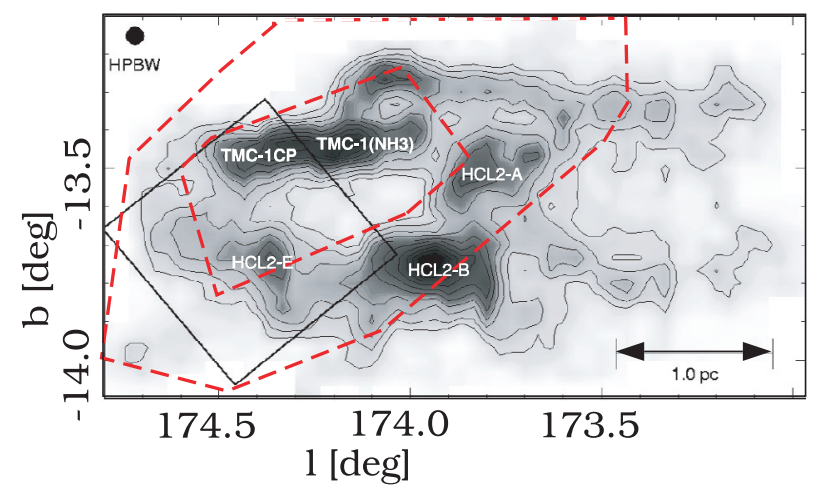

Fig. 9. HCL2 with TMR and its main clouds. $\mathrm{C}^{18} \mathrm{O}$ line intensity $W\left(\mathrm{C}^{18} \mathrm{O}\right)$ distribution as observed by the Nagoya-4 $\mathrm{m}$ telescope $\left(H P B W=2.7\right.$ shown in the upper left corner) in a $2^{\prime}$ grid. The $W\left(\mathrm{C}^{18} \mathrm{O}\right)$ contours (thin continuous lines) are drawn from $0.33 \mathrm{~K} \mathrm{~km}^{-1}$ by $0.1 \mathrm{~K} \mathrm{~km} \mathrm{~s}^{-1}$. The apparent $W\left(\mathrm{C}^{18} \mathrm{O}\right)$ maxima are at TMC-1 (top left), HCL2-E (left), HCL2-B (center down), HCL2-A (center). The area of the ISOPHOT image is marked with a black frame. We represent the associated $\mathrm{HI}$ cloud with $\mathrm{HI} 21 \mathrm{~cm}$ line area contours of $341 \mathrm{~K} \mathrm{~km} \mathrm{~s}^{-1}$ and $352 \mathrm{~K} \mathrm{~km} \mathrm{~s}^{-1}$ (red dashed line).

generally follows the distribution of visual extinction. According to Fig. 4a of Wilson \& Mink (1977) the primary maximum of the column density of cold $\mathrm{HI}$ coincides with the position of the ISOPHOT cold clouds HCL2E/HCL2ES. The TMR hole is near the HI peak and no drop was seen in the HI column density with the $9^{\prime}$ resolution of the Effelsberg$100 \mathrm{~m}$ telescope; however, the TMR hole is approximately $15^{\prime} \times 10^{\prime}$ in size. The average cold HI column density is $N(\mathrm{HI})_{\text {cold }} \approx 0.8 \frac{T_{\text {spin }}}{3.2 \mathrm{~K}} \times 10^{19} \mathrm{~cm}^{-2}$ and the total cold HI mass in HCL2 is $M(\mathrm{HI}) \approx \frac{T_{\text {spin }}}{3.2 \mathrm{~K}} \times 8 M_{\odot}$ (after Wilson \& Mink 1977). Both values depend on the assumed $T_{\text {spin }}$ excitation temperature. The estimated mass of $\mathrm{HI}$ gas in HCL2 is $M(\mathrm{HI}) \approx 25 M_{\odot}$. The total molecular mass of HCL2 is over $800 M_{\odot}$ (Onishi et al. 1996), we thus interpret TMR as series of molecular condensations in a massive cloud with $>95 \%$ of its hydrogen content in molecular form.

Dynamics of TMR TMR with $\approx 90 \%$ of the mass of HCL2, an effective radius of $r_{\text {eff }}(\mathrm{TMR})=1.5 \mathrm{pc}$ and a velocity dispersion of $\delta v \approx 1.5 \mathrm{~km} \mathrm{~s}^{-1}$ is gravitationally bound. We consider TMR as a torus that looks elliptical with an apparent axial ratio of 0.6 at $\approx 40^{\circ}$ inclination. Schloerb \& Snell (1984) and Heyer (1988) proposed that the ISM circulates in TMR, based on their ${ }^{13} \mathrm{CO}$ data. We did not find a velocity gradient along the apparent major axis in our $\mathrm{C}^{18} \mathrm{O}$ spectra. A clear velocity difference is seen between the NE and NW parts of TMR south in $\mathrm{C}^{18} \mathrm{O}$ lines, with the $\mathrm{NW}$ part being $0.5 \mathrm{~km} \mathrm{~s}^{-1}$ more redshifted. Accordingly we may assume that TMR is contracting.

A magnetic field is present in TMR as seen in well aligned $K$-band polarization, with a mean position angle of about $50 \mathrm{deg}$ (Tamura et al. 1987). This is perpendicular to the direction of the elongation of the HCL2 cloud, which may indicate a formation by contraction along the magnetic field lines. That theory alone however does not explain the presence of the hole. After all, the structure of TMR can be interpreted as well as a couple of dense clouds arranged into two filaments, 
with a gap between them. Some of the simulated CO spectra and maps of Stenholm \& Pudritz (1993) resembles to TMR. This means that an initially uniform, gravitating and radiating cloud with thermal and magnetic pressure (the latter due to a spectrum of Alfvénic waves) may look like TMR after 3.5 free fall times. We note also the remarkable differences between the sub-clouds (or in other words fragments) of TMR. As we found, TMC-1 CP and HCL2-E in the southern part of TMR are without bright IRAS pointsoures (however still showing star formation), while HCL-B in the northern part has a group of them.

Williams \& Hartquist (1991) claimed that interaction between stellar winds and molecular gas causes a cyclic interchange between high and low density phases. The differences between the cloudlets of HCL2 is then explained by the different "interaction history", and age. If there is an age gradient in TMR, it is from north to south. The southern part of TMR (the target of this paper) is evolutionary the youngest in TMR, with HCL2-ES in an assumed pre-core-formation phase. An age gradient of TMC-1 has already been proposed by several authors, Saito et al. (2002) recently claimed $10^{5} \mathrm{yr}$ as a possible age difference in TMC-1 between the ammonia peak and the cyanopolyyne peak.

Padoan et al. (2002) conclude that the observed column density distribution is very likely the result of turbulent fragmentation. Hartmann (2002) however explains the filamentary structures of the Taurus clouds as a result of converging large scale flows channeled by magnetic fields. A detailed numerical simulation is required to illustrate how the large scale effects gave rise to the density peaks in a ring and not simply in the middle of the HI cloud. It should show if such a structure can also be formed in a turbulent cloud.

\section{Conclusions}

- The $200 \mu \mathrm{m}$ ISO image of HCL2 by ISOPHOT resolved the dense southern part of the Taurus Molecular Ring (TMR) as a complex of cold clouds.

- The brightest $200 \mu \mathrm{m}$ objects of the southern part of TMR correspond to the cores of TMC-1 CP and HCL2-E, both of which have dust colour temperatures $\leq 12 \mathrm{~K}$, like the coldest cloud cores of the nearby ISM.

- The dust colour temperatures and gas kinetic temperatures are similar in the cloud cores.

- The cores have densities of $n(\mathrm{H}) \approx 10^{5} \mathrm{~cm}^{-3}$.

- There is one new YSO and several new YSO candidates found towards the southern TMR.

- The $\mathrm{C}^{18} \mathrm{O}$ kinematics of TMR does not support the rotating ring theory, but rather reflects two filaments of clouds or a collapsing ring structure.

Acknowledgements. We thank P. Padoan and L. Cambresy for providing us with their $A_{\mathrm{V}}$ data of Taurus. We also thank our referee A. Jones for his comments. The ISOPHOT Data Centre at MPIA is supported by Deutsches Zentrum für Luft und Raumfahrt (DLR) with funds of the Bundesministerium für Bildung und Forschung, grant No. 50QI0201. This research was partly supported by a grant of the Academy of Finland No. 174854. and an OTKA grant No. T034998. Results are based also on observations with the 100-m telescope of the
MPIfR (Max-Planck-Institut für Radioastronomie) at Effelsberg. This research has made use of the NASA/IPAC Infrared Science Archive, which is operated by the Jet Propulsion Laboratory, California Institute of Technology, under contract with the National Aeronautics and Space Administration. This publication makes use of data products from the Two Micron All Sky Survey, which is a joint project of the University of Massachusetts and the Infrared Processing and Analysis Center/California Institute of Technology, funded by the National Aeronautics and Space Administration and the National Science Foundation.

\section{References}

Abergel, A., Boulanger, F., Mizuno, A., \& Fukui, Y. 1994, ApJ, 423, 59

Abergel, A., Boulanger, F., Mizuno, A., \& Fukui, Y. 1995, A\&AS, 111,483

Baars, J. W. M., Genzel, R., Pauliny-Toth, I. I. K., \& Witzel, A. 1977, A\&A, 61, 99

Briceno, C., Calvet, N., Kenyon, S., \& Hartmann, L. 1999, AJ, 118, 1354

Brown, D. W., \& Chandler, C. J. 1999, MNRAS, 303, 855

Cao, Y., Terebey, S., Prince, T. A., \& Beichman, C. A. 1997, ApJS, 111,387

Cernicharo, J., Guèlin, M., \& Askne, J. 1984, A\&A, 138, 371

del Burgo, C., Laureijs, R. J., Ábrahám, P., \& Kiss, Cs. 2003, MNRAS, 346,403

Elias, J. H. 1978, ApJ, 224, 875

Harju, J., Walmsley, C. M., \& Wouterloot, J. G. A. 1993, A\&AS, 98, 51

Harju, J., Winnberg, A., \& Wouterloot, J. G. A. 2000, A\&A, 353, 1065

Hartigan, P., \& Kenyon, S. J. 2003, ApJ, 583, 334

Hartmann, L. 2002, ApJ, 578, 914

Hartmann, D., \& Burton, W. B. 1999, VizieR On-line Data Catalog: VIII/54

Harvey, D. W. A., Wilner, D. J., Di Francesco, J., et al. 2002, AJ, 123, 3325

Heiles, C. E. 1968, ApJ, 151, 919

Heyer, M. H. 1988, ApJ, 324, 311

Helou, G., \& Kahn, I. 1986, All About SCANPI, IPAC memo.

Hirahara, Y., Suzuki, H., Yamamoto, S., et al. 1992, ApJ, 394, 539

Itoh, Y., Tamura, M., \& Gatley, I. 1996, ApJ, 465, L129

Itoh, Y., Tamura, M., \& Nakajima, T. 1999, AJ, 117, 1471

Itoh, Y., Tamura, M., \& Tokunaga, A. T. 2002, PASJ, 54, 561

Juvela, M., Mattila, K., Lehtinen, K., et al. 2002, A\&A, 382, 583

Klaas, U., Laureijs, R., Radovich, M., \& Schulz, B. 1998, ISOPHOT Calibration Accuracies, SAI/98-092/Dc Version 2.0 in ISO Explanatory Library

Koenig, B., Neuhauser, R., \& Selzer, B. 2001, A\&A, 369, 971

Laureijs, R. J., Clark, F. O., \& Prusti, T. 1991, ApJ, 372, 185

Lagache, G., Abergel, A., Boulanger, F., et al. 1998, A\&A, 333, 709

Langer, W. D., Velusamy, T., Kuiper, T. B., et al. 1995, ApJ, 453, 293

Lee, C. W., Myers, P. C., \& Taffala, M. 2001, ApJS, 136, 703

Lemke, D., Klaas, U., Abolins, J., et al. 1996, A\&A, 315, L64

Little, L. T., MacDonald, G. H., Riley, P. W., \& Matheson, D. N. 1979, MNRAS, 189, 539

Lynds, B. T. 1962, ApJS, 7, 1

Markwick, A. J., Millar, T. J., \& Charnley, S. B. 2000, ApJ, 535, 256

Mizuno, A., Onishi, T., Yonekura, Y., et al. 1995, ApJ, 445, L161

Murphy, D. C., \& Myers, P. C. 2003, ApJ, 591, 1034

Olano, C. A., Walmsley, C. M., \& Wilson, T. L. 1988, A\&A, 196, 194

Onishi, T., Mizuno, A., Kawamura, A., et al. 1996, ApJ, 465, 815 
Onishi, T., Mizuno, A., Kawamura, A., Ogawa, H., \& Fukui, Y. 1998, Tamura, M., Nagata, T., Sato, S., \& Tanaka, M. 1987, MNRAS, 224, ApJ, 502, 296 413

Onishi, T., Mizuno, A., Kawamura, A., Tachihara, K., \& Fukui, Y. 2002, ApJ, 575, 950

Padoan, P., Cambrésy, L., \& Langer, W. 2002, ApJ, 580, 57

Park, S., \& Kenyon, S. J. 2002, AJ, 123, 3370

Peng, R., Langer, W. D., Velusamy, T. B. H., et al. 1998, ApJ, 497, 842

Rydgren, A. E., \& Vrba, F. J. 1983, AJ, 88, 1017

Stenholm, L. G., \& Pudritz, R. E. 1993, ApJ, 416, 218

Takakuwa, S., Mikami, H., Saito, M., \& Hirano, N. 2000, ApJ, 542, 367

Tóth, L. V., Vavrek, R., \& Lemke, D. 2004, Baltic Astronomy, in press Voges, W., Aschenbach, B., Boller, Th., et al. 1999, A\&A, 349, 389

Weintraub, D. A. 1990, ApJS, 74, 575

Williams, J. P., Blitz, L., \& McKee, C. F. 1999, Protostars and Planets IV, 97

Schlegel, D. J., Finkbeiner, D. P., \& Davis, M. 1998, ApJ, 500, 525

Schloerb, F. P., \& Snell, R. L. 1984, ApJ, 283, 129

Saito, S., Aikawa, Y., \& Herbst, E. 2002, ApJ, 569, 836

Williams, D. A., \& Hartquist, T. W. 1991, MNRAS, 251, 351

Willacy, K., Langer, W. D., \& Velusamy, T. 1998, ApJ, 507, 171

Wilson, T. L., \& Minn, Y. K. 1977, A\&A, 54, 933

Whitworth, A. P., Boffin, H. M. J., \& Francis, N. 1998, ASP Conf. Ser., 183

Stepnik, B., Abergel, A., Bernard, J.-P., et al. 2003, A\&A, 398, Ward-Thompson, D., Scott, P. F., Hills, R. E., \& Andre, P. 1994, 551 MNRAS, 268, 276 